AperTO - Archivio Istituzionale Open Access dell'Università di Torino

The Neural Correlates of Time: A Meta-analysis of Neuroimaging Studies

This is a pre print version of the following article:

Original Citation:

Availability:

This version is available http://hdl.handle.net/2318/1723185

since 2020-01-15T15:24:42Z

Published version:

DOI:10.1162/jocn_a_01459

Terms of use:

Open Access

Anyone can freely access the full text of works made available as "Open Access". Works made available under a Creative Commons license can be used according to the terms and conditions of said license. Use of all other works requires consent of the right holder (author or publisher) if not exempted from copyright protection by the applicable law. 


\title{
The Neural Correlates of Time: A Meta-analysis of Neuroimaging Studies
}

\author{
Andrea Nani ${ }^{1,2}$, Jordi Manuello ${ }^{1,2}$, Donato Liloia ${ }^{1,2}$, Sergio Duca ${ }^{1,2}$, \\ Tommaso Costa ${ }^{1,2}$, and Franco Cauda ${ }^{1,2}$
}

\begin{abstract}
During the last two decades, our inner sense of time has been repeatedly studied with the help of neuroimaging techniques. These investigations have suggested the specific involvement of different brain areas in temporal processing. At least two distinct neural systems are likely to play a role in measuring time: One is mainly constituted by subcortical structures and is supposed to be more related to the estimation of time intervals below the 1-sec range (subsecond timing tasks), and the other is mainly constituted by cortical areas and is supposed to be more related to the estimation of time intervals above the 1-sec range (suprasecond timing tasks). Tasks can then be performed in motor or nonmotor (perceptual) conditions, thus providing four different categories of time processing. Our
\end{abstract}

\section{INTRODUCTION}

The passage of time is one of the most pervasive aspects of our lives. Intuitively, everyone feels to know what it means that time passes, but if we were asked to express in words the nature of time and the feeling of temporality, we would be in the same situation as Saint Augustine, who declared in his Confessions: "What then is time? If no one asks me, I know what it is. If I wish to explain it to him who asks, I do not know." Saint Augustine wrote between the IV and V centuries $\mathrm{AD}$, but to date the nature of time remains elusive. Time has been considered an essential feature of reality, on the one hand (Unger \& Smolin, 2015), or a figment of the way we know reality, on the other (Rovelli, 2018). Independent of the fact that time is a real constituent of the outside world or just a fabrication of our inner world, the brain is unquestionably involved in perceiving or constructing the temporal framework within which events (seem to) happen and interact.

During the last two decades, time perception (or construction) has been repeatedly investigated with the help of neuroimaging techniques. These studies have put forward different results, which in turn have been used to support different theories about the involvement

${ }^{1}$ GCS-fMRI, Koelliker Hospital, Turin, Italy, ${ }^{2}$ University of Turin meta-analytical investigation partly confirms the findings of previous meta-analytical works. Both sub- and suprasecond tasks recruit cortical and subcortical areas, but subcortical areas are more intensely activated in subsecond tasks than in suprasecond tasks, which instead receive more contributions from cortical activations. All the conditions, however, show strong activations in the SMA, whose rostral and caudal parts have an important role not only in the discrimination of different time intervals but also in relation to the nature of the task conditions. This area, along with the striatum (especially the putamen) and the claustrum, is supposed to be an essential node in the different networks engaged when the brain creates our sense of time.

of specific brain areas in temporal processing. These theories can be divided into two types: dedicated and intrinsic models (Ivry \& Schlerf, 2008). The dedicated models hypothesize a specific mechanism exclusively devoted to the estimation of time; in contrast, the intrinsic models assume that various brain structures normally associated with sensory and cognitive processes can also act as interval timers (Wittmann, 2013). A classic example of a dedicated mechanism for time perception is the pacemaker-accumulator system (Taatgen, van Rijn, \& Anderson, 2007; Zakay \& Block, 1997). The idea is that the neural pacemaker generates a series of pulses that, just like the ticks of a clock, are recorded to measure time intervals. In particular, the dopaminergic system and the BG, especially the striatum (caudate nucleus and putamen) and the substantia nigra pars compacta, are thought to be the underpinnings of this neural pacemaker (Meck, Penney, \& Pouthas, 2008; Buhusi \& Meck, 2005). In particular, the striatum, which receives inputs from a large number of cortical neurons, would be the main region involved in detecting the oscillatory patterns or frequencies related to the activity of different cortical sites. Neuroimaging investigations that applied different techniques (fMRI, PET) have supported the pivotal role played by the striatum in time processing (Harrington, Zimbelman, Hinton, \& Rao, 2010; Coull \& Nobre, 2008; Rao, Mayer, \& Harrington, 2001). However, other experiments provide evidence that, according to different tasks 
and conditions, other several brain areas are supposed to be involved in the perception of time.

Various mechanisms underlying intrinsic models have therefore been proposed. The most general mechanism assumes that short-term synaptic plasticity, which is at the basis of the dynamics of neural states, can transform the brain networks in time keepers (Buonomano \& Maass, 2009). According to this view, every cerebral area has the capacity to process and estimate time. Other proposals, on the contrary, are more specific and pinpoint a certain brain region or neural population as the substrate of a particular temporal task. For instance, the right $\mathrm{pFC}$ (Lewis \& Miall, 2006a, 2006b), the cerebellum (Ivry, Spencer, Zelaznik, \& Diedrichsen, 2002), the SMA (Macar, Coull, \& Vidal, 2006), the right inferior parietal lobule (Battelli, Pascual-Leone, \& Cavanagh, 2007), and the anterior insula (Craig, 2009a) have been variously associated with time perception, judgment, and the processing of intervals' duration.

The variegated picture of our inner sense of time created by the involvement of these various brain structures is probably due to different experimental contexts and settings, the characteristics of the tasks employed, the duration of stimuli, the nature of responses, as well as the application of different statistical procedures. For example, Lewis and Miall (2003a) carried out a label-based review of neuroimaging studies on time perception. The authors divided the experimental results according to the interval duration (i.e., subsecond or suprasecond tasks), the use of movement (i.e., motor or nonmotor tasks) and the nature of response (i.e., automatic or cognitively controlled tasks).

With regard to motor timing research, the task that is most frequently used is paced finger tapping: Participants are asked to tap a key following the rhythm of an isochronous metronome and then continue tapping at the same pace in absence of stimulation. In some experiments, the continuation phase is not required, but the participants are asked to tap at the middle point between groups of pacing stimuli. Other nonmotor experiments use the interval reproduction task, in which participants recall the duration of an interval between two stimuli to which they were previously exposed. A variation of this task is to ask the participants directly, without exposure, to produce a specific interval (i.e., "produce an interval of $6 \mathrm{sec}$ "). On the other hand, time perception tasks require participants to judge the span of intervals. Frequently, participants are asked to compare and discriminate the duration of two successive stimuli by judging which of them is longer than the other. In these experiments, the duration of the stimuli to be judged is very variable, ranging from 200 to $24 \mathrm{sec}$. According to these different timing tasks, different brain structures are recruited (Buhusi \& Meck, 2005; Mauk \& Buonomano, 2004; Lewis \& Miall, 2003a). Furthermore, neuropsychological studies in humans and research on animals point out that the estimation of temporal intervals above and below $1 \mathrm{sec}$ relies on the activation of different neural mechanisms and assemblies (Harrington \& Haaland, 1999; Gibbon, Malapani, Dale, \& Gallistel, 1997).

Overall, with regard to these different experimental conditions, studies have suggested that at least two distinct neural systems are likely to play a role in measuring time: One is mainly constituted by subcortical structures and is supposed to be more related to subsecond timing tasks, and the other is mainly constituted by cortical areas and is supposed to be more related to suprasecond timing tasks.

The distinction between different tasks and time intervals has been also highlighted by the meta-analysis of Wiener, Turkeltaub, and Coslett (2010), which analyzed four different conditions: subsecond motor, subsecond perceptual (i.e., nonmotor), suprasecond motor, and suprasecond perceptual. We have decided to adhere to this distinction, as it describes better the current literature on time perception studies. It should be observed, however, that it has some limitations, especially with regard to the category of the "perceptual" condition. In fact, rigorously speaking, this category is not strictly perceptual, as its experimental tasks include also the engagement of higher order cognitive functions, such as attention and salience. As Wiener et al. (2010), we use therefore "perceptual" in a broad sense as a synonym of "nonmotor," so as to designate all the experiments in which the task requirement is not strictly motor. For the studies that tested an interval across subsecond and suprasecond stimuli, we also decided, like Wiener et al. (2010), to determine whether most of the interval was above or below $1 \mathrm{sec}$ and attribute them to the corresponding category. Furthermore, with regard to these possible confounding factors, we applied the fail-safe techinque to assess the robustness of our results (see Methods for a detailed explanation of this procedure).

The results of the meta-analysis by Wiener et al. (2010) supported the idea that certain neural structures are involved in subsecond timing tasks and others in suprasecond timing tasks. In particular, the analysis of subsecond tasks revealed a greater activation of subcortical sites, such as the bilateral cerebellum and left BG. In contrast, the analysis of suprasecond tasks principally showed the activations of cortical areas, such as the bilateral SMA, frontal cortex, and cingulate gyri. Similarly, the meta-analysis carried out by Ortuño, Guillen-Grima, Lopez-Garcia, Gomez, and Pla (2011) largely reproduced these findings. However, in this case statistical analyses were performed on independent studies and only considered the cerebral activations related to implicit or explicit time estimation tasks. Finally, Schwartze, Rothermich, and Kotz (2012) focused their meta-analysis of fMRI studies on a subarea of the SMA by contrasting different temporal processing functions, such as sensory, sensorimotor, sequential, nonsequential, explicit, nonexplicit, subsecond, or suprasecond tasks. These authors identified different activations of the SMA, which might be correlated with different types of temporal processing. 
Table 1. Articles Included in the Meta-analysis Using the PRISMA Statement International Guidelines

\begin{tabular}{|c|c|c|c|c|c|}
\hline Study & Tool & Participants & Foci & $\begin{array}{c}\text { Experimental Task } \\
\text { (Contrast or Condition) }\end{array}$ & Duration (msec) \\
\hline Aso et al. (2010) (Exp. A) & fMRI & 14 & 6 & $\begin{array}{l}\text { Temporal reproduction (main } \\
\text { effect of motor timing) }\end{array}$ & $500-999$ \\
\hline Bengtsson et al. (2005) & fMRI & 7 & 6 & $\begin{array}{l}\text { Paced finger tapping and } \\
\text { articulation (more vs. less } \\
\text { complex) }\end{array}$ & $375-750-1125$ \\
\hline $\begin{array}{l}\text { Bueti, Walsh, et al. } \\
\text { (2008) (Exp. A) }\end{array}$ & fMRI & 14 & 11 & $\begin{array}{l}\text { Temporal reproduction } \\
\text { (action vs. control) }\end{array}$ & $300-600-900-1200$ \\
\hline Berns et al. (1997) & PET & 10 & 3 & $\begin{array}{l}\text { RT sequence (more vs. } \\
\text { less complex) }\end{array}$ & 700 \\
\hline $\begin{array}{l}\text { Jahanshahi et al. } \\
\text { (2006) (Exp. A) }\end{array}$ & PET & 8 & 11 & $\begin{array}{l}\text { Temporal reproduction } \\
\text { (short vs. long intervals) }\end{array}$ & 500 \\
\hline Jancke et al. (2000) & fMRI & 8 & 3 & $\begin{array}{l}\text { Paced finger tapping } \\
\quad \text { (paced vs. cued finger) }\end{array}$ & 400 \\
\hline Jantzen et al. (2004) & fMRI & 14 & 10 & $\begin{array}{l}\text { Paced finger tapping (syncopation } \\
\text { vs. synchronization) }\end{array}$ & 800 \\
\hline Jantzen et al. (2005) & fMRI & 12 & 7 & $\begin{array}{l}\text { Paced finger tapping (syncopation } \\
\text { vs. synchronization) }\end{array}$ & 800 \\
\hline Jantzen et al. (2007) & fMRI & 9 & 7 & $\begin{array}{l}\text { Paced finger tapping (paced vs. } \\
\text { cued finger) }\end{array}$ & 800 \\
\hline Karabanov et al. (2009) & fMRI & 16 & 43 & $\begin{array}{l}\text { Temporal sequence performance } \\
\text { (visual vs. auditory rhythms) }\end{array}$ & $375-750-1125-1500$ \\
\hline Lewis et al. (2004) & fMRI & 10 & 42 & $\begin{array}{l}\text { Paced finger tapping (paced vs. } \\
\text { cued finger) }\end{array}$ & 700 \\
\hline Lutz et al. (2000) & fMRI & 14 & 1 & $\begin{array}{l}\text { Paced finger tapping (isochronous } \\
\text { vs. nonisochronous) }\end{array}$ & 667 \\
\hline Mayville et al. (2002) & fMRI & 9 & 0 & $\begin{array}{l}\text { Paced finger tapping (syncopation } \\
\text { vs. synchronization) }\end{array}$ & 800 \\
\hline $\begin{array}{l}\text { Murai \& Yotsumoto } \\
\text { (2016) (Exp. A) }\end{array}$ & fMRI & 21 & 7 & $\begin{array}{l}\text { Temporal duration reproduction } \\
\text { (sub vs. suprasecond) }\end{array}$ & 400 \\
\hline Ortuño et al. (2002) & & 10 & 9 & $\begin{array}{l}\text { Temporal articulation (mentally vs. } \\
\text { auditory rhythm counting) }\end{array}$ & 1000 \\
\hline $\begin{array}{l}\text { Oullier et al. (2005) } \\
\text { (Exp. A) }\end{array}$ & fMRI & 15 & 19 & $\begin{array}{l}\text { Paced finger tapping (syncopation } \\
\text { vs. synchronization) }\end{array}$ & 800 \\
\hline $\begin{array}{l}\text { Oullier et al. (2005) } \\
\quad \text { (Exp. B) }\end{array}$ & fMRI & 15 & 12 & $\begin{array}{l}\text { Paced finger tapping (synchronization } \\
\text { vs. syncopation) }\end{array}$ & 800 \\
\hline Pastor et al. (2004) & fMRI & 14 & 6 & $\begin{array}{l}\text { Temporal discrimination } \\
\text { (clicks vs. location) }\end{array}$ & $5-140$ \\
\hline $\begin{array}{l}\text { Penhune et al. } \\
\text { (1998) (Exp. A) }\end{array}$ & PET & 11 & 5 & $\begin{array}{l}\text { Paced finger tapping (novel } \\
\text { vs. learned) }\end{array}$ & 250 \\
\hline $\begin{array}{l}\text { Penhune et al. } \\
\text { (1998) (Exp. B) }\end{array}$ & PET & 11 & 4 & $\begin{array}{l}\text { Paced finger tapping (novel } \\
\text { vs. learned) }\end{array}$ & 750 \\
\hline Pope et al. (2005) & fMRI & 13 & 21 & $\begin{array}{l}\text { Temporal sequences reproduction } \\
\text { (main effect of alternating force) }\end{array}$ & $400-800$ \\
\hline $\begin{array}{l}\text { Rao et al. (1997) } \\
\quad \text { (Exp. A) }\end{array}$ & fMRI & 13 & 7 & $\begin{array}{l}\text { Temporal discrimination } \\
\text { (temporal continuation vs. pitch) }\end{array}$ & 300 \\
\hline
\end{tabular}


Table 1. (continued)

\begin{tabular}{|c|c|c|c|c|c|}
\hline Study & Tool & Participants & Foci & $\begin{array}{c}\text { Experimental Task } \\
\text { (Contrast or Condition) }\end{array}$ & Duration (msec) \\
\hline Rao et al. (1997) (Exp. B) & fMRI & 13 & 7 & $\begin{array}{l}\text { Temporal discrimination } \\
\text { (temporal continuation vs. pitch) }\end{array}$ & 600 \\
\hline $\begin{array}{l}\text { Schubotz \& von Cramon } \\
\text { (2001) (Exp. A) }\end{array}$ & fMRI & 12 & 20 & $\begin{array}{l}\text { Temporal reproduction (temporal } \\
\text { vs. control pressing) }\end{array}$ & $290-1450$ \\
\hline \multicolumn{6}{|c|}{ Subsecond motor timing condition: 21 articles, 24 experiments, 293 participants, and 287 foci of activation } \\
\hline $\begin{array}{l}\text { Aso et al. (2010) } \\
\qquad \text { (Exp. B) }\end{array}$ & fMRI & 14 & 4 & $\begin{array}{l}\text { Temporal discrimination } \\
\quad \text { (main effect for sensory timing) }\end{array}$ & 500-999 \\
\hline $\begin{array}{l}\text { Bengtsson et al. } \\
\text { (2009) (Exp. A) }\end{array}$ & fMRI & 17 & 12 & $\begin{array}{l}\text { Auditory rhythm perception } \\
\text { (rhythm vs. random) }\end{array}$ & $200-400-600-800$ \\
\hline $\begin{array}{l}\text { Bengtsson et al. } \\
\text { (2009) (Exp. B) }\end{array}$ & fMRI & 17 & 7 & $\begin{array}{l}\text { Auditory rhythm perception } \\
\text { (metric vs. isochronous) }\end{array}$ & 200-300-700-900 \\
\hline $\begin{array}{l}\text { Beudel et al. } \\
\text { (2009) (Exp. A) }\end{array}$ & fMRI & 18 & 10 & $\begin{array}{l}\text { Temporal estimation (time } \\
\text { ahead vs. stop) }\end{array}$ & $100-800$ \\
\hline $\begin{array}{l}\text { Beudel et al. } \\
\text { (2009) (Exp. B) }\end{array}$ & fMRI & 18 & 9 & $\begin{array}{l}\text { Temporal estimation (time } \\
\text { ahead vs. spatial place) }\end{array}$ & $100-800$ \\
\hline $\begin{array}{l}\text { Bueti, Walsh, et al. } \\
\text { (2008) (Exp. B) }\end{array}$ & fMRI & 14 & 4 & $\begin{array}{l}\text { Temporal estimation (perception } \\
\text { vs. control) }\end{array}$ & $300-600-900-1200$ \\
\hline $\begin{array}{l}\text { Coull \& Nobre } \\
\qquad(1998) \text { (Exp. A) }\end{array}$ & PET & 15 & 4 & $\begin{array}{l}\text { Temporal perception (when } \\
\text { vs. where) }\end{array}$ & $0-315$ \\
\hline $\begin{array}{l}\text { Coull \& Nobre } \\
\text { (1998) (Exp. B) }\end{array}$ & fMRI & 15 & 3 & $\begin{array}{l}\text { Temporal perception (when } \\
\text { vs. where) }\end{array}$ & $0-315$ \\
\hline $\begin{array}{l}\text { Ferrandez et al. } \\
\text { (2003) }\end{array}$ & fMRI & 11 & 12 & $\begin{array}{l}\text { Temporal discrimination (estimation } \\
\text { vs. intensity of duration) }\end{array}$ & 700 \\
\hline $\begin{array}{l}\text { Gutyrchik et al. } \\
\text { (2010) }\end{array}$ & fMRI & 13 & & $\begin{array}{l}\text { Temporal perception (duration } \\
\text { vs. color) }\end{array}$ & $100-250-1000-1300$ \\
\hline $\begin{array}{l}\text { Lewis \& Miall } \\
\qquad \text { (2003b) (Exp. A) }\end{array}$ & fMRI & & 20 & $\begin{array}{l}\text { Temporal discrimination } \\
\text { (temporal vs. length) }\end{array}$ & 600 \\
\hline Maquet et al. (1996) & PET & 9 & 6 & $\begin{array}{l}\text { Temporal discrimination } \\
\text { (time vs. intensity duration) }\end{array}$ & 700 \\
\hline Mathiak et al. (2004) & fMRI & 12 & 1 & $\begin{array}{l}\text { Temporal auditory discrimination } \\
\text { (auditory tones duration) }\end{array}$ & 200 \\
\hline Neufang et al. (2008) & fMRI & 34 & 1 & $\begin{array}{l}\text { Temporal discrimination (time vs. } \\
\text { visual compatibility) }\end{array}$ & $250-350$ \\
\hline Schubotz et al. (2000) & fMRI & 20 & 14 & $\begin{array}{l}\text { Temporal discrimination (temporal } \\
\text { vs. color pitch) }\end{array}$ & 900 \\
\hline $\begin{array}{l}\text { Schubotz \& von Cramon } \\
\text { (2001) (Exp. B) }\end{array}$ & fMRI & 12 & 20 & $\begin{array}{l}\text { Temporal monitoring (temporal vs. } \\
\text { control monitoring) }\end{array}$ & $290-1450$ \\
\hline Shih et al. (2009) & fMRI & 17 & 5 & $\begin{array}{l}\text { Temporal discrimination } \\
\text { (temporal vs. control) }\end{array}$ & $100-450$ \\
\hline Shih et al. (2010) & fMRI & 21 & 13 & $\begin{array}{l}\text { Temporal discrimination } \\
\text { (temporal vs. control) }\end{array}$ & $400-450$ \\
\hline Tipples et al. (2013) & fMRI & 17 & 8 & $\begin{array}{l}\text { Temporal estimation (duration vs. } \\
\text { gender of stimuli) }\end{array}$ & $400-700-1000-1300-1600$ \\
\hline Tomasi et al. (2015) & fMRI & 36 & 27 & Temporal prediction (temporal vs. & 600 \\
\hline
\end{tabular}


Table 1. (continued)

\begin{tabular}{|c|c|c|c|c|c|}
\hline Study & Tool & Participants & Foci & $\begin{array}{l}\text { Experimental Task } \\
\text { (Contrast or Condition) }\end{array}$ & Duration (msec) \\
\hline \multicolumn{6}{|c|}{ memory attention) } \\
\hline Tregellas et al. (2006) & fMRI & 20 & 10 & $\begin{array}{l}\text { Temporal discrimination } \\
\text { (difficult vs. easy) }\end{array}$ & 200 \\
\hline $\begin{array}{l}\text { van Wassenhove et al. } \\
\text { (2011) (Exp. A) }\end{array}$ & fMRI & 15 & 6 & $\begin{array}{l}\text { Temporal dilation estimation } \\
\quad \text { (looming vs. standard stimuli) }\end{array}$ & 500 \\
\hline $\begin{array}{l}\text { van Wassenhove et al. } \\
\text { (2011) (Exp. B) }\end{array}$ & fMRI & 15 & 8 & $\begin{array}{l}\text { Temporal dilation estimation } \\
\quad \text { (receding vs. standard stimuli) }\end{array}$ & 500 \\
\hline $\begin{array}{l}\text { Wittmann, van Wassenhove, } \\
\text { et al. (2010) (Exp. A) }\end{array}$ & fMRI & 20 & 6 & $\begin{array}{l}\text { Temporal dilation estimation } \\
\text { (looming vs. standard stimuli) }\end{array}$ & 500 \\
\hline $\begin{array}{l}\text { Wittmann, van Wassenhove, } \\
\text { et al. (2010) (Exp. B) }\end{array}$ & fMRI & 20 & 8 & $\begin{array}{l}\text { Temporal dilation estimation } \\
\text { (receding vs. standard stimuli) }\end{array}$ & 500 \\
\hline \multicolumn{6}{|c|}{ Subsecond perceptual (nonmotor) timing condition: 20 articles, 25 experiments, 428 participants, and 220 foci of activation } \\
\hline Ackermann et al. (2001) & fMRI & 8 & 9 & $\begin{array}{l}\text { Paced finger tapping (isochronous trains } \\
\text { of clicks at different frequencies) }\end{array}$ & 6000 \\
\hline Basso et al. (2003) & fMRI & 5 & 8 & $\begin{array}{l}\text { Temporal production (time vs. } \\
\text { working memory control) }\end{array}$ & 1500 \\
\hline $\begin{array}{l}\text { Botzung et al. (2008) } \\
\quad \text { (Exp. A) }\end{array}$ & fMRI & 10 & 11 & $\begin{array}{l}\text { Events evocation (past vs. semantic } \\
\text { memory condition) }\end{array}$ & $3700-11240$ \\
\hline $\begin{array}{l}\text { Botzung et al. (2008) } \\
\quad \text { (Exp. B) }\end{array}$ & fMRI & 10 & 12 & $\begin{array}{l}\text { Events evocation (future vs. semantic } \\
\text { memory condition) }\end{array}$ & $3700-11240$ \\
\hline Brunia et al. (2000) & PET & 8 & 4 & $\begin{array}{l}\text { Temporal production (correct vs. } \\
\text { incorrect feedback) }\end{array}$ & 3000 \\
\hline $\begin{array}{l}\text { Coull et al. (2013) } \\
\text { (Exp. A) }\end{array}$ & fMRI & 27 & & $\begin{array}{l}\text { Temporal production (temporal vs. } \\
\text { neutral reproduction) }\end{array}$ & $600-1000-1400$ \\
\hline $\begin{array}{l}\text { Dudukovic \& Wagner } \\
\text { (2007) }\end{array}$ & fMRI & 18 & 11 & $\begin{array}{l}\text { Temporal decision (recent vs. } \\
\text { new stimuli) }\end{array}$ & 5000 \\
\hline Garraux et al. (2005) & fMRI & 1 & 1 & Paced finger tapping (timing vs. order) & 2000 \\
\hline $\begin{array}{l}\text { Jahanshahi et al. (2006) } \\
\text { (Exp. B) }\end{array}$ & PI & 8 & 14 & $\begin{array}{l}\text { Temporal reproduction (long vs. } \\
\text { short intervals) }\end{array}$ & 2000 \\
\hline Jech et al. (2005) & fMRI & 9 & 4 & $\begin{array}{l}\text { Temporal reproduction (increase } \\
\text { in duration length) }\end{array}$ & $500-16800$ \\
\hline Kawashima et al. (2000) & fMRI & 8 & 4 & $\begin{array}{l}\text { Paced finger tapping (paced vs. } \\
\text { cued finger) }\end{array}$ & 1500 \\
\hline Knutson et al. (2004) & fMRI & 19 & 7 & $\begin{array}{l}\text { Temporal order (script vs. } \\
\text { chronological order) }\end{array}$ & 2500 \\
\hline Larsson et al. (1996) & fMRI & 8 & 4 & $\begin{array}{l}\text { Paced finger tapping (paced vs. } \\
\text { cued finger) }\end{array}$ & 3800 \\
\hline Lejeune et al. (1997) & PET & 12 & 4 & $\begin{array}{l}\text { Paced finger tapping (paced vs. } \\
\text { cued finger) }\end{array}$ & 2700 \\
\hline Lewis \& Miall (2002) & fMRI & 8 & 9 & $\begin{array}{l}\text { Temporal reproduction (reproduction } \\
\text { vs. control pressing) }\end{array}$ & 3000 \\
\hline Macar et al. (2002) & PET & 15 & 6 & $\begin{array}{l}\text { Temporal reproduction (temporal vs. } \\
\text { control pressing) }\end{array}$ & $2200-3200-9000-13000$ \\
\hline Macar et al. (2004) & fMRI & 13 & 2 & Temporal reproduction & 2500 \\
\hline
\end{tabular}


Table 1. (continued)

\begin{tabular}{|c|c|c|c|c|c|}
\hline Study & Tool & Participants & Foci & $\begin{array}{c}\text { Experimental Task } \\
\text { (Contrast or Condition) }\end{array}$ & Duration (msec) \\
\hline \multicolumn{6}{|c|}{ (temporal vs. force) } \\
\hline $\begin{array}{l}\text { Murai \& Yotsumoto } \\
\text { (2016) (Exp. B) }\end{array}$ & fMRI & 21 & 16 & $\begin{array}{l}\text { Temporal duration reproduction } \\
\text { (supra vs. subsecond) }\end{array}$ & 2500 \\
\hline $\begin{array}{l}\text { Rekkas et al. (2005) } \\
\quad \text { (Exp. A) }\end{array}$ & fMRI & 10 & 3 & $\begin{array}{l}\text { Temporal discrimination (semantic } \\
\text { temporal order vs. spatial location) }\end{array}$ & 6000 \\
\hline $\begin{array}{l}\text { Rekkas et al. (2005) } \\
\quad \text { (Exp. B) }\end{array}$ & fMRI & 10 & 5 & $\begin{array}{l}\text { Temporal discrimination (autobiographic } \\
\text { temporal order vs. spatial location) }\end{array}$ & 6000 \\
\hline Riecker et al. (2003) & fMRI & 8 & 8 & $\begin{array}{l}\text { Paced finger tapping (isochronous trains } \\
\text { of clicks at different frequencies) }\end{array}$ & 6000 \\
\hline Riecker et al. (2006) & fMRI & 8 & 13 & $\begin{array}{l}\text { Paced finger tapping (self-paced syllable } \\
\text { repetition at different frequencies) }\end{array}$ & 6000 \\
\hline Rubia et al. (1998) & fMRI & 6 & 4 & $\begin{array}{l}\text { Paced finger tapping (high- vs. } \\
\text { low-frequency synchronization) }\end{array}$ & 3000 \\
\hline Shergill et al. (2006) & fMRI & 8 & 9 & Temporal articulation (paced vs. cued) & 2000 \\
\hline Stevens et al. (2007) & fMRI & 31 & 12 & $\begin{array}{l}\text { Paced finger tapping (syncopation } \\
\text { vs. synchronization) }\end{array}$ & $750-1500-3500$ \\
\hline Taniwaki et al. (2006) & fMRI & 12 & 16 & $\begin{array}{l}\text { Temporal execution (self-initiated vs. } \\
\text { externally triggered movements) }\end{array}$ & 40000 \\
\hline Teki \& Griffiths (2016) & fMRI & 19 & 9 & $\begin{array}{l}\text { Temporal reproduction (temporal } \\
\text { vs. control) }\end{array}$ & $1500-2000$ \\
\hline Tracy et al. (2000) & fMRI & 6 & 3 & $\begin{array}{l}\text { Temporal production (duration } \\
\text { reproduction at different intervals) }\end{array}$ & $1200-2400$ \\
\hline $\begin{array}{l}\text { Wittmann, Simmons, } \\
\text { et al. (2010) (Exp. A) }\end{array}$ & fMRI & 14 & 3 & $\begin{array}{l}\text { Temporal reproduction (temporal } \\
\text { vs. control) }\end{array}$ & 3000 \\
\hline $\begin{array}{l}\text { Wittmann, Simmons, } \\
\text { et al. (2010) (Exp. B) }\end{array}$ & fMRI & 14 & & $\begin{array}{l}\text { Temporal reproduction (temporal } \\
\text { vs. control) }\end{array}$ & 9000 \\
\hline $\begin{array}{l}\text { Wittmann, Simmons, } \\
\text { et al. (2010) (Exp. C) }\end{array}$ & fMRI & 14 & 3 & $\begin{array}{l}\text { Temporal reproduction (temporal } \\
\text { vs. control) }\end{array}$ & 18000 \\
\hline $\begin{array}{l}\text { Wittmann et al. } \\
\text { (2011) (Exp. A) }\end{array}$ & fMRI & 27 & 8 & $\begin{array}{l}\text { Temporal reproduction (temporal } \\
\text { vs. control) }\end{array}$ & 3000 \\
\hline $\begin{array}{l}\text { Wittmann et al. } \\
\text { (2011) (Exp. B) }\end{array}$ & fMRI & 27 & 4 & $\begin{array}{l}\text { Temporal reproduction (temporal } \\
\text { vs. control) }\end{array}$ & 9000 \\
\hline $\begin{array}{l}\text { Wittmann et al. } \\
\text { (2011) (Exp. C) }\end{array}$ & fMRI & 27 & 6 & $\begin{array}{l}\text { Temporal reproduction (temporal } \\
\text { vs. control) }\end{array}$ & 18000 \\
\hline \multicolumn{6}{|c|}{ Suprasecond motor timing condition: 28 articles, 34 experiments, 459 participants, and 261 foci of activation } \\
\hline Apaydın et al. (2018) & fMRI & 18 & 9 & $\begin{array}{l}\text { Temporal perception (time } \\
\text { vs. reward) }\end{array}$ & 2500 \\
\hline $\begin{array}{l}\text { Carvalho et al. } \\
\text { (2016) (Exp. A) }\end{array}$ & fMRI & 16 & 16 & $\begin{array}{l}\text { Temporal expectation (nonperiodic } \\
\text { vs. periodic) }\end{array}$ & $1340-2000$ \\
\hline $\begin{array}{l}\text { Carvalho et al. } \\
\text { (2016) (Exp. B) }\end{array}$ & fMRI & 16 & 7 & $\begin{array}{l}\text { Temporal expectation (nonperiodic } \\
\text { vs. periodic) }\end{array}$ & $1340-2000$ \\
\hline Coull et al. (2000) & fMRI & 6 & 18 & $\begin{array}{l}\text { Temporal estimation (long vs. } \\
\text { short target presentation) }\end{array}$ & $600-1400$ \\
\hline Coull et al. (2004) & fMRI & 12 & 10 & Temporal discrimination (increase & 540-1080-1620 \\
\hline
\end{tabular}


Table 1. (continued)

\begin{tabular}{|c|c|c|c|c|c|}
\hline Study & Tool & Participants & Foci & $\begin{array}{c}\text { Experimental Task } \\
\text { (Contrast or Condition) }\end{array}$ & Duration (msec) \\
\hline & & & & in attention to time) & \\
\hline $\begin{array}{l}\text { Coull et al. (2013) } \\
\text { (Exp. B) }\end{array}$ & fMRI & 27 & 15 & $\begin{array}{l}\text { Temporal prediction (temporal vs. } \\
\text { neutral orienting) }\end{array}$ & $600-1000-1400$ \\
\hline Dirnberger et al. (2012) & fMRI & 7 & 7 & $\begin{array}{l}\text { Incorrect time estimation (aversive } \\
\text { vs. neutral context) }\end{array}$ & $1800-2200$ \\
\hline Harrington et al. (2004) & fMRI & 24 & 26 & $\begin{array}{l}\text { Temporal duration discrimination } \\
\text { (main effect of encoding) }\end{array}$ & $1200-1800$ \\
\hline Harrington et al. (2010) & fMRI & 20 & 7 & $\begin{array}{l}\text { Temporal duration discrimination } \\
\quad \text { (time vs. pitch) }\end{array}$ & $10000-12500$ \\
\hline Herrmann et al. (2014) & fMRI & 19 & 5 & $\begin{array}{l}\text { Temporal rate change perception } \\
\text { (sound vs. silent trials) }\end{array}$ & 6400 \\
\hline Jueptner et al. (1996) & fMRI & 6 & 6 & $\begin{array}{l}\text { Temporal discrimination (temporal } \\
\text { vs. constant velocity) }\end{array}$ & 1000 \\
\hline $\begin{array}{l}\text { Lewis \& Miall (2003b) } \\
\quad \text { (Exp. B) }\end{array}$ & fMRI & 8 & 13 & $\begin{array}{l}\text { Temporal discrimination (temporal } \\
\text { vs. length) }\end{array}$ & 3000 \\
\hline Livesey et al. (2007) & fMRI & 10 & 4 & $\begin{array}{l}\text { Temporal discrimination (temporal } \\
\text { vs. color) }\end{array}$ & $1000-1500$ \\
\hline Lux et al. (2003) & fMRI & 14 & 5 & $\begin{array}{l}\text { Temporal estimation (temporal } \\
\text { synchrony vs. spatial orientation) }\end{array}$ & 1050 \\
\hline Morillon et al. (2009) & fMRI & 27 & 17 & $\begin{array}{l}\text { Temporal estimation (duration } \\
\text { vs. color) }\end{array}$ & $100-8400$ \\
\hline Pfeuty et al. (2015) & fMRI & 29 & 6 & $\begin{array}{l}\text { Temporal estimation (duration } \\
\text { vs. color) }\end{array}$ & 1500 \\
\hline Pouthas et al. (2005) & fMRI & 6 & 6 & $\begin{array}{l}\text { Temporal estimation (long vs. } \\
\text { short duration) }\end{array}$ & 1300 \\
\hline $\begin{array}{l}\text { Rao et al. (2001) } \\
\quad \text { (Exp. A) }\end{array}$ & fMRI & 17 & & $\begin{array}{l}\text { Temporal discrimination (temporal } \\
\text { perception vs. control) }\end{array}$ & 2500 \\
\hline $\begin{array}{l}\text { Rao et al. (2001) } \\
\quad \text { (Exp. B) }\end{array}$ & fMRI & 17 & 3 & $\begin{array}{l}\text { Temporal discrimination (temporal } \\
\text { perception vs. control) }\end{array}$ & 5000 \\
\hline $\begin{array}{l}\text { Rao et al. (2001) } \\
\quad \text { (Exp. C) }\end{array}$ & $\mathrm{fMR}$ & 17 & 10 & $\begin{array}{l}\text { Temporal discrimination (temporal } \\
\text { perception vs. control) }\end{array}$ & 75000 \\
\hline $\begin{array}{l}\text { Rao et al. (2001) } \\
\quad \text { (Exp. D) }\end{array}$ & fMRI & 17 & 9 & $\begin{array}{l}\text { Temporal discrimination (temporal } \\
\text { perception vs. control) }\end{array}$ & 10000 \\
\hline Smith et al. (2003) & fMRI & 20 & 7 & $\begin{array}{l}\text { Temporal discrimination (temporal } \\
\text { vs. order) }\end{array}$ & 1000 \\
\hline Smith et al. (2011) & fMRI & 32 & 3 & $\begin{array}{l}\text { Temporal discrimination (temporal } \\
\text { vs. order) }\end{array}$ & 2100 \\
\hline $\begin{array}{l}\text { Tsukamoto et al. } \\
\text { (2006) }\end{array}$ & fMRI & 20 & 13 & $\begin{array}{l}\text { Temporal estimation (true vs. false } \\
\text { feedback condition) }\end{array}$ & 3000 \\
\hline Ustun et al. (2017) & fMRI & 15 & 10 & $\begin{array}{l}\text { Temporal discrimination } \\
\text { (time vs. visual) }\end{array}$ & $2400-6000$ \\
\hline Wiener et al. (2014) & fMRI & 25 & 17 & $\begin{array}{l}\text { Temporal discrimination } \\
\text { (time vs. color) }\end{array}$ & 2000 \\
\hline
\end{tabular}


Figure 1. PRISMA flow chart. Overview of the selection strategy.
So, although interesting meta-analyses have been published in the last 15 years, substantial disagreement about the dynamics of functional activations of the brain structures associated with time processing still persists. Furthermore, some methodological innovations support the choice to carry out a novel meta-analysis on time perception, as the last works on this topic used an old version of GingerALE software (Ortuño et al., 2011; Wiener et al., 2010). In fact, after 2016 the BrainMap team reported some technical errors affecting this tool. In particular, codes for the thresholding procedure with both false discovery rate and cluster-level family-wise error methods were not working as expected, so that the results could be biased by false positives (for a detailed discussion, see Eickhoff, Laird, Fox, Lancaster, \& Fox, 2017). In particular, Eickhoff et al. (2016) have explicitly discouraged the use of false discovery rate, in virtue of a high susceptibility to false positive results. These authors have recommended the implementation of cluster-level family-wise error thresholding, which was used in the present work, along with the new GingerALE version in which the errors of the algorithm have been corrected. Furthermore, previous meta-analyses have worked on a number of studies that were far smaller than ours. Wiener et al. (2010) examined 41 neuroimaging studies, Schwartze et al. (2012) examined 42 fMRI studies, and Ortuño et al. (2011) examined
35 studies; in contrast, this meta-analysis examined 84 studies, thus basically doubling the samples of the past.

\section{METHODS}

\section{Selection of Studies}

The MEDLINE database was queried to identify all published experiments on time perception or processing that utilized functional neuroimaging tool (fMRI or PET). Using the search engine PubMed (https://www.ncbi. nlm.nih.gov/pubmed/), we adopted a MeSH hierarchy routine. Furthermore, we used the function [ALL] of PubMed to find MeSH terms in all the fields of the eligible articles. The search algorithm was constructed as follows:

(time perception OR [ALL] time estimation OR [ALL] time processing [ALL]) AND (functional magnetic resonance OR [ALL] fMRI OR [ALL] positron emission tomography OR [ALL] PET [ALL]).

Additionally, references from previous review articles (Teki, 2016; Merchant, Harrington, \& Meck, 2013) and metaanalyses (Schwartze et al., 2012; Ortuño et al., 2011; Wiener et al., 2010) about time perception were reviewed for inclusion. At the time of the selection phase (June 2018), over 170 
full-text articles were assessed for eligibility. In particular, two expert researchers included in the meta-analysis the studies on the basis of the following criteria:

a) articles were published in a peer-reviewed journal;

b) articles included only healthy participants;

c) articles adopted a whole-brain analysis (not just ROIs);

d) articles reported coordinates of activation in Talairach/ Tourneaux or in Montreal Neurological Institute stereotactic brain space;

e) articles included at least one contrast of timing task (not contrast task-rest).

We therefore included in our meta-analysis 84 published articles, for a total of 109 experiments, 1018 coordinates of activation, and 1218 healthy participants (see Table 1 for detailed information about the sample). To facilitate the subsequent analysis, we converted all native Montreal Neurological Institute coordinates into Talairach/ Tourneaux brain space using the icbm2tal transformation (Laird et al., 2010).

Following the previous studies of Lewis and Miall (2003a) and Wiener et al. (2010), our data set was partitioned into two main dimensions: interval duration (i.e., subsecond and suprasecond) and type (i.e., motor and nonmotor) of experimental time estimation task. Accordingly, we carried out our meta-analysis on four distinct samples: subsecond motor (287 coordinates and 24 experiments), subsecond nonmotor (220 coordinates and 25 experiments), suprasecond motor (261 coordinates and 34 experiments), and suprasecond nonmotor (250 coordinates and 26 experiments; see also Table 1). In this data set, we included all the 41 studies considered by Wiener et al. (2010) and 21 studies — also included by Wiener et al. (2010)—of 28 of those considered by Lewis and Miall (2003a). Seven studies were excluded because one was an EEG study, one was a PET study on rhesus monkeys, four had rest contrast, and one had no dissociation between sub- and suprasecond tasks. The entire process of data selection was performed using the Preferred Reporting Items for Systematic Reviews and Meta-analyses (PRISMA) Statement international guidelines (Liberati et al., 2009; Moher, vLiberati, Tetzlaff, \& Altman, 2009; for the overview of the selection strategy, see Figure 1).

\section{Activation Likelihood Estimation and Modeled Alteration Creation}

To statistically summarize the results of the retrieved experiments for each of the four conditions previously identified, we performed an activation likelihood estimation

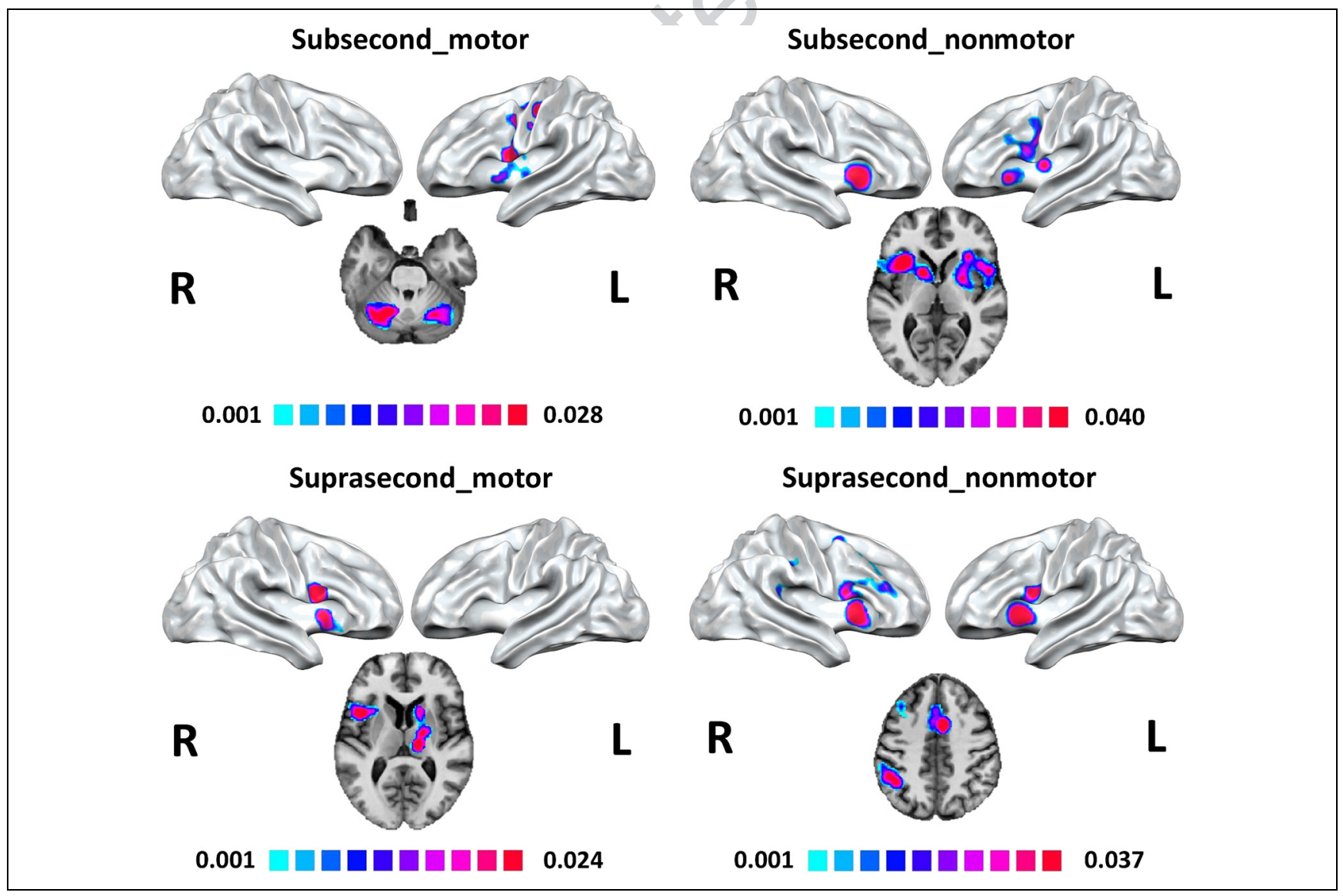

Figure 2. Results of the ALE analyses for the four conditions (cluster level corrected, $p<.05$ ). Colors from light blue to red represent increasing ALE values. Slices on the axial plane are shown in radiological convention (i.e., right is left, left is right). 
Table 2. Significant ALE Results for the Subsecond Motor Condition Meta-analysis

\begin{tabular}{|c|c|c|c|c|c|c|c|}
\hline \multirow[b]{2}{*}{ Cluster } & \multirow[b]{2}{*}{ Volume $\left(\mathrm{mm}^{3}\right)$} & \multirow[b]{2}{*}{ Label } & \multirow[b]{2}{*}{ Side } & \multirow{2}{*}{$\begin{array}{c}\text { Extrema } \\
\text { Value }\end{array}$} & \multicolumn{3}{|c|}{ Talairach } \\
\hline & & & & & $x$ & $y$ & $z$ \\
\hline \multirow[t]{21}{*}{1} & 34,248 & Medial frontal gyrus (BA 6) & $\mathrm{L}$ & 0.028 & -6 & -4 & 52 \\
\hline & & Medial frontal gyrus (BA 6) & $\mathrm{R}$ & 0.024 & 4 & -4 & 60 \\
\hline & & Medial frontal gyrus (BA 6) & $\mathrm{R}$ & 0.022 & 8 & 4 & 56 \\
\hline & & Precentral gyrus (BA 6) & $\mathrm{L}$ & 0.022 & -56 & 0 & 20 \\
\hline & & Precentral gyrus (BA 4) & $\mathrm{L}$ & 0.019 & -36 & -22 & 56 \\
\hline & & Superior frontal gyrus (BA 6) & $\mathrm{L}$ & 0.018 & -2 & 6 & 48 \\
\hline & & Subgyral (BA 6) & $\mathrm{L}$ & 0.016 & -24 & -4 & 52 \\
\hline & & Middle frontal gyrus (BA 6) & $\mathrm{L}$ & 0.013 & -26 & -10 & 42 \\
\hline & & Cingulate gyrus (BA 32) & $\mathrm{R}$ & 0.012 & 10 & 10 & 36 \\
\hline & & Precentral gyrus (BA 6) & $\mathrm{L}$ & 0.012 & -44 & 0 & 48 \\
\hline & & Insula (BA 13) & $\mathrm{L}$ & 0.011 & -42 & -10 & 16 \\
\hline & & Postcentral gyrus (BA 3) & $\mathrm{L}$ & 0.009 & -48 & -16 & 44 \\
\hline & & Superior temporal gyrus (BA 22) & $\mathrm{L}$ & 0.009 & -52 & 6 & 4 \\
\hline & & Precentral gyrus (BA 6) & $\mathrm{L}$ & 0.009 & -14 & -16 & 66 \\
\hline & & Precentral gyrus (BA 44) & $\mathrm{L}$ & 0.008 & -50 & 0 & 8 \\
\hline & & Superior frontal gyrus (BA 6) & $\mathrm{R}$ & 0.007 & 12 & 16 & 62 \\
\hline & & Precentral gyrus (BA 6) & $\mathrm{L}$ & 0.007 & -48 & -2 & 34 \\
\hline & & Superior frontal gyrus (BA 6) & $\mathrm{L}$ & 0.007 & -8 & 8 & 70 \\
\hline & & Middle frontal gyrus (BA 6) & $\mathrm{L}$ & 0.007 & -56 & 2 & 40 \\
\hline & & Inferior parietal lobule (BA 40 & $\mathrm{~L}$ & 0.007 & -40 & -36 & 44 \\
\hline & & Inferior frontal gyrus (BA 45) & $\mathrm{L}$ & 0.006 & -52 & 18 & 6 \\
\hline \multirow[t]{4}{*}{2} & 12,352 & Ventral posterior medial nucleus (thalamus) & $\mathrm{L}$ & 0.028 & -14 & -20 & 6 \\
\hline & & Lentiform nucleus (putamen) & $\mathrm{L}$ & 0.022 & -26 & -8 & 6 \\
\hline & & Claustrum & $\mathrm{L}$ & 0.008 & -30 & 10 & 2 \\
\hline & & Claustrum & $\mathrm{L}$ & 0.006 & -32 & -8 & -8 \\
\hline \multirow[t]{9}{*}{3} & 11,752 & Lentiform nucleus (putamen) & $\mathrm{R}$ & 0.018 & 20 & 4 & 12 \\
\hline & & Lentiform nucleus (putamen) & $\mathrm{R}$ & 0.017 & 20 & -2 & 10 \\
\hline & & Substania nigra & $\mathrm{R}$ & 0.015 & 10 & -20 & -6 \\
\hline & & Lentiform nucleus (putamen) & $\mathrm{R}$ & 0.015 & 24 & -12 & 6 \\
\hline & & Medial dorsal nucleus (thalamus) & $\mathrm{R}$ & 0.014 & 10 & -18 & 6 \\
\hline & & Caudate body & $\mathrm{R}$ & 0.011 & 20 & 12 & 14 \\
\hline & & Claustrum & $\mathrm{R}$ & 0.008 & 26 & 14 & 16 \\
\hline & & Insula (BA 13) & $\mathrm{R}$ & 0.007 & 30 & 16 & 16 \\
\hline & & Ventral posterior lateral nucleus (thalamus) & $\mathrm{R}$ & 0.007 & 24 & -24 & 10 \\
\hline
\end{tabular}


Table 2. (continued)

\begin{tabular}{|c|c|c|c|c|c|c|c|}
\hline \multirow[b]{2}{*}{ Cluster } & \multirow[b]{2}{*}{ Volume $\left(\mathrm{mm}^{3}\right)$} & \multirow[b]{2}{*}{ Label } & \multirow[b]{2}{*}{ Side } & \multirow{2}{*}{$\begin{array}{c}\text { Extrema } \\
\text { Value }\end{array}$} & \multicolumn{3}{|c|}{ Talairach } \\
\hline & & & & & $x$ & $y$ & $z$ \\
\hline \multirow[t]{6}{*}{4} & 9512 & Culmen (cerebellum) & $\mathrm{R}$ & 0.019 & 14 & -52 & -18 \\
\hline & & Culmen (cerebellum) & $\mathrm{R}$ & 0.019 & 38 & -50 & -30 \\
\hline & & Declive (cerebellum) & $\mathrm{R}$ & 0.014 & 28 & -60 & -22 \\
\hline & & Tonsil (cerebellum) & $\mathrm{R}$ & 0.009 & 28 & -42 & -30 \\
\hline & & Tonsil (cerebellum) & $\mathrm{R}$ & 0.006 & 20 & -58 & -34 \\
\hline & & Declive (cerebellum) & $\mathrm{R}$ & 0.005 & 2 & -60 & -16 \\
\hline \multirow[t]{9}{*}{5} & 9336 & Culmen (cerebellum) & $\mathrm{L}$ & 0.012 & -22 & -56 & -24 \\
\hline & & Tonsil (cerebellum) & $\mathrm{L}$ & 0.012 & -34 & -42 & -44 \\
\hline & & Declive (cerebellum) & $\mathrm{L}$ & 0.011 & -30 & -58 & -18 \\
\hline & & Tonsil (cerebellum) & $\mathrm{L}$ & 0.010 & -24 & -58 & -44 \\
\hline & & Uvula (cerebellum) & $\mathrm{L}$ & 0.010 & -34 & -62 & -26 \\
\hline & & Tonsil (cerebellum) & $\mathrm{L}$ & 0.009 & -28 & -52 & -34 \\
\hline & & Tonsil (cerebellum) & L & 0.007 & -38 & -58 & -44 \\
\hline & & Declive (cerebellum) & $\mathrm{L}$ & 0.007 & -12 & -64 & -18 \\
\hline & & Pyramis (cerebellum) & , & 0.007 & -44 & -64 & -32 \\
\hline
\end{tabular}

\section{Contributors to Cluster 1}

11 foci from Karabanov et al. (2009)

10 foci from Lewis et al. (2004)

6 foci from Schubotz \& von Cramon (2001) (Exp. A)

5 foci from Pope et al. (2005)

4 foci from Ortuño et al. (2002)

3 foci from Murai \& Yotsumoto (2016) (Exp. A)

3 foci from Jantzen et al. (2005)

3 foci from Pastor et al. (2004)

3 foci from Oullier et al. (2005) (Exp. A)

4 foci from Oullier et al. (2005) (Exp. B)

\section{Contributors to Cluster 2}

4 foci from Lewis et al. (2004)

3 foci from Mayville et al. (2002)

3 foci from Karabanov et al. (2009)

2 foci from Schubotz \& von Cramon (2001) (Exp. A)

2 foci from Rao et al. (1997) (Exp. A)

3 foci from Rao et al. (1997) (Exp. B)

2 foci from Pope et al. (2005)
2 foci from Jantzen et al. (2007)

2 foci from Jantzen et al. (2004)

2 foci from Jancke et al. (2000)

2 foci from Rao et al. (1997) (Exp. A)

3 foci from Rao et al. (1997) (Exp. B)

2 foci from Bueti et al. (2008) (Exp. A)

1 focus from Berns et al. (1997)

1 focus from Bengtsson et al. (2005)

1 focus from Penhune et al. (1998) (Exp. B)

1 focus from Jantzen et al. (2005)

1 focus from Jantzen et al. (2004)

1 focus from Penhune et al. (1998) (Exp. A)

1 focus from Oullier et al. (2005) (Exp. A)

1 focus from Oullier et al. (2005) (Exp. B)

1 focus from Bueti et al. (2008) (Exp. A) 
Table 2. (continued)

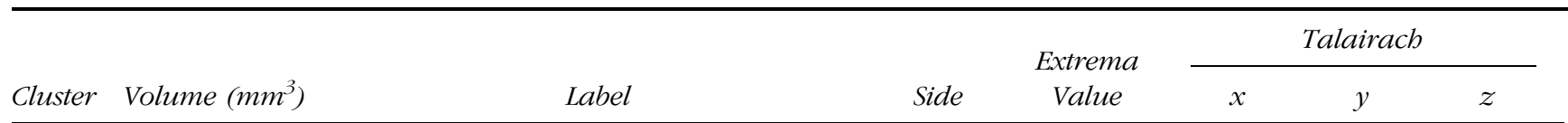

Contributors to Cluster 3

6 foci from Mayville et al. (2002)

6 foci from Karabanov et al. (2009)

2 foci from Lewis et al. (2004)

2 foci from Schubotz \& von Cramon (2001) (Exp. A)

2 foci from Pope et al. (2005)

\section{Contributors to Cluster 4}

3 foci from Bengtsson et al. (2005)

3 foci from Karabanov et al. (2009)

2 foci from Lewis et al. (2004)

2 foci from Mayville et al. (2002)

1 focus from Jantzen et al. (2004)

1 focus from Schubotz \& von Cramon (2001) (Exp. A)

Contributors to Cluster 5

2 foci from Schubotz \& von Cramon (2001) (Exp. A)

2 foci from Karabanov et al. (2009)

2 foci from Aso et al. (2010) (Exp. A)

1 focus from Lewis et al. (2004)

1 focus from Jantzen et al. (2007)

1 focus from Jantzen et al. (2005)

1 focus from Jantzen et al. (2004)
2 foci from Oullier et al. (2005) (Exp. A)

2 foci from Oullier et al. (2005) (Exp. A)

2 foci from Bueti et al. (2008) (Exp. A)

2 foci from Jantzen et al. (2004)

For each cluster brain regions, cluster size $\left(\mathrm{mm}^{3}\right)$, side (hemisphere), ALE value, Talairach coordinates, and contributors to cluster (foci) were provided. $\mathrm{BA}=$ Brodmann's area; $\mathrm{L}=$ left; $\mathrm{R}=$ right.

(ALE) as implemented in GingerAle 2.3.6 (Eickhoff, Bzdok, Laird, Kurth, \& Fox, 2012; Turkeltaub et al., 2012; Eickhoff et al., 2009). The four resulting maps have been clustered at a level of $p<.05$, family-wise error-corrected for multiple comparisons, with a cluster-forming threshold of $p<.001$ (Eickhoff et al., 2016, 2017).

The ALE is a technique capable of providing information about the reliability of results by means of a comparison between the experiments and a sample of reference studies from the existing literature (Laird et al., 2005). An ALE meta-analysis considers every experiment focus as being a Gaussian probability distribution:

$$
p(d)=\frac{1}{\sigma^{3} \sqrt{(2 \pi)^{3}}} e-\frac{d^{2}}{2 \sigma^{2}}
$$

1 focus from Rao et al. (1997) (Exp. A)

1 focus from Rao et al. (1997) (Exp. B)

1 focus from Pope et al. (2005)

1 focus from Oullier et al. (2005) (Exp. A)

1 focus from Ortuño et al. (2002)

1 focus from Bueti et al. (2008) (Exp. A)
1 focus from Pope et al. (2005)

1 focus from Penhune et al. (1998) (Exp. A)

1 focus from Penhune et al. (1998) (Exp. B)

1 focus from Oullier et al. (2005) (Exp. A)

1 focus from Oullier et al. (2005) (Exp. B)

1 focus from Ortuño et al. (2002)

1 focus from Bueti et al. (2008) (Exp. A) in which $d$ is the Euclidean distance between the focus and the voxels whereas is the standard deviation of the distribution.

A modeled alteration map is then determined for every experiment as the combination of the Gaussian probability distribution of every experiment focus. Subsequently, the ALE map is obtained from the union of all the modeled alteration maps.

The ALE map is thresholded on the basis of a null hypothesis, obtained recursively by producing all the possible random distribution of foci. Then, to obtain a cluster-level correction, a similar procedure is repeated to define the size of nonrandom clusters of activation. The significance of values within the ALE map is finally calculated by testing the null hypothesis that active voxels are randomly distributed across the brain. The iterative 
Table 3. Significant ALE Results for the Subsecond Perceptual Condition Meta-analysis

\begin{tabular}{|c|c|c|c|c|c|c|c|}
\hline \multirow[b]{2}{*}{ Cluster } & \multirow[b]{2}{*}{ Volume $\left(\mathrm{mm}^{3}\right)$} & \multirow[b]{2}{*}{ Label } & \multirow[b]{2}{*}{ Side } & \multirow[b]{2}{*}{ Extrema Value } & \multicolumn{3}{|c|}{ Talairach } \\
\hline & & & & & $x$ & $y$ & $z$ \\
\hline \multirow[t]{16}{*}{1} & 25,192 & Claustrum & $\mathrm{L}$ & 0.031 & -30 & -6 & 12 \\
\hline & & Inferior frontal gyrus (BA 47) & $\mathrm{L}$ & 0.022 & -48 & 12 & 0 \\
\hline & & Claustrum & $\mathrm{L}$ & 0.020 & -28 & 24 & 4 \\
\hline & & Precentral gyrus (BA 44) & $\mathrm{L}$ & 0.019 & -46 & 8 & 12 \\
\hline & & Lentiform nucleus (putamen) & $\mathrm{L}$ & 0.017 & -22 & 2 & 8 \\
\hline & & Precentral gyrus (BA 6) & $\mathrm{L}$ & 0.016 & -46 & 0 & 32 \\
\hline & & Middle frontal gyrus (BA 6) & $\mathrm{L}$ & 0.015 & -24 & -8 & 50 \\
\hline & & Inferior frontal gyrus (BA 9) & $\mathrm{L}$ & 0.015 & -52 & 10 & 26 \\
\hline & & Insula (BA 13) & $\mathrm{L}$ & 0.014 & -40 & 14 & 6 \\
\hline & & Lentiform nucleus (putamen) & $\mathrm{L}$ & 0.014 & -24 & 10 & 4 \\
\hline & & Precentral gyrus (BA 6) & $\mathrm{L}$ & 0.009 & -48 & -2 & 8 \\
\hline & & Middle frontal gyrus (BA 6) & $\mathrm{L}$ & 0.009 & -38 & 2 & 46 \\
\hline & & Middle frontal gyrus (BA 9) & $\mathrm{L}$ & 0.008 & -48 & 22 & 32 \\
\hline & & Middle frontal gyrus (BA 46) & $\mathrm{L}$ & 0.008 & -46 & 22 & 20 \\
\hline & & Middle frontal gyrus (BA 6) & $L$ & 0.007 & -48 & 4 & 48 \\
\hline & & Middle frontal gyrus (BA 9) & $\mathrm{L}$ & 0.006 & -46 & 32 & 38 \\
\hline \multirow[t]{5}{*}{2} & 14,896 & Insula (BA 13) & & 0.032 & 34 & 18 & 6 \\
\hline & & Caudate body & $\mathrm{R}$ & 0.022 & 14 & 8 & 6 \\
\hline & & Insula (BA 13) & $\mathrm{R}$ & 0.014 & 44 & 10 & 16 \\
\hline & & Superior temporal gyrus (BA 22) & $\mathrm{R}$ & 0.011 & 50 & 12 & 0 \\
\hline & & Lentiform nucleus (globus pallidus) & $\mathrm{R}$ & 0.010 & 20 & -2 & -6 \\
\hline \multirow[t]{5}{*}{3} & 13,208 & Cingulate gyrus (BA 32) & $\mathrm{L}$ & 0.040 & 0 & 28 & 34 \\
\hline & & Superior frontal gyrus (BA 6) & $\mathrm{R}$ & 0.023 & 2 & 10 & 50 \\
\hline & & Medial frontal gyrus (BA 6) & $\mathrm{L}$ & 0.016 & -2 & -4 & 54 \\
\hline & & Cingulate gyrus (BA 32) & $\mathrm{L}$ & 0.008 & -12 & 6 & 40 \\
\hline & & Medial frontal gyrus (BA 6) & $\mathrm{L}$ & 0.008 & -4 & -18 & 52 \\
\hline
\end{tabular}

Contributors to Cluster 1

6 foci from Tomasi et al. (2015)

5 foci from Schubotz \& von Cramon (2001) (Exp. B)

4 foci from Lewis \& Miall (2003b)

4 foci from Ferrandez et al. (2003)

3 foci from Tregellas et al. (2006)

3 foci from Schubotz et al. (2000)

2 foci from Beudel et al. (2009) (Exp. A)
2 foci from Shih et al. (2009)

2 foci from Bueti et al. (2008)

1 focus from Wittmann et al. (2010a) (Exp. A)

1 focus from Wittmann et al. (2010a) (Exp. C)

1 focus from van Wassenhove et al. (2011) (Exp. A)

1 focus from van Wassenhove et al. (2011) (Exp. B)

1 focus from Coull \& Nobre (1998) (Exp. A) 
Table 3. (continued)

\begin{tabular}{|c|c|c|c|c|c|c|}
\hline \multirow[b]{2}{*}{ Cluster } & \multirow[b]{2}{*}{ Volume $\left(\mathrm{mm}^{3}\right)$} & \multirow[b]{2}{*}{ Side } & \multirow[b]{2}{*}{ Extrema Value } & \multicolumn{3}{|c|}{ Talairach } \\
\hline & & & & $x$ & $y$ & $z$ \\
\hline 2 foci fr & m Bengtsson et al. (2009) (Exp. A) & \multicolumn{5}{|c|}{1 focus from Beudel et al. (2009) (Exp. B) } \\
\hline 2 foci fr & m Tipples et al. (2013) & \multicolumn{5}{|c|}{1 focus from Aso et al. (2010) (Exp. B) } \\
\hline
\end{tabular}

\section{Contributors to Cluster 2}

5 foci from Tomasi et al. (2015)

3 foci from Lewis \& Miall (2003b)

3 foci from Tipples et al. (2013)

3 foci from Schubotz \& von Cramon (2001) (Exp. B)

2 foci from Tregellas et al. (2006)

2 foci from Schubotz et al. (2000)

1 focus from Wittmann et al. (2010a) (Exp. B)

1 focus from van Wassenhove et al. (2011) (Exp. B)
1 focus from Shih et al. (2010)

1 focus from Ferrandez et al. (2003)

1 focus from Beudel et al. (2009) (Exp. A)

2 focus from Beudel et al. (2009) (Exp. B)

1 focus from Maquet et al. (1996)

1 focus from Bueti et al. (2008) (Exp. B)

1 focus from Aso et al. (2010) (Exp. B)

\section{Contributors to Cluster 3}

3 foci from Bengtsson et al. (2009) (Exp. A)

2 foci from Lewis \& Miall, 2003b

2 foci from Ferrandez et al. (2003)

2 foci from Tipples et al. (2013)

2 foci from Shih et al. (2009)

2 foci from Schubotz \& von Cramon (2001) (Exp. B)

1 focus from Wittmann et al. (2010a) (Exp. A)

1 focus from Wittmann et al. (2010a) (Exp. B)

1 focus from van Wassenhove et al. (2011) (Exp. A)
1 focus from van Wassenhove et al. (2011) (Exp. B)

1 focus from Tomasi et al. (2015)

1 focus from Gutyrchik et al. (2010)

1 focus from Beudel et al. (2009) (Exp. A)

1 focus from Beudel et al. (2009) (Exp. B)

1 focus from Tregellas et al. (2006)

1 focus from Schubotz et al. (2000)

1 focus from Maquet et al. (1996)

For each cluster brain regions, cluster size $\left(\mathrm{mm}^{3}\right)$, side (hemisphere), ALE value, Talairach coordinates, and contributors to cluster (foci) were provided. $\mathrm{BA}=$ Brodmann's area; $\mathrm{L}=$ left; $\mathrm{R}=$ right.

process generates a vector that can be considered as a histogram representing the null distribution. Voxel-level thresholds can be obtained from this. The cluster-level threshold is obtained through the calculation of the cluster size distribution, after 1000 permutations (Acar, Seurinck, Eickhoff, \& Moerkerke, 2018).

\section{Correlation between Maps and Voxels' Contribution to Correlation Analysis}

To compare the four different conditions, we computed all the possible correlations between the maps. To do so, we computed the linear correlation, by means of Pearson's $r$, between the two matrices containing the voxel values of the map associated with each condition.
For every condition, we further analyzed the voxelwise contribution to the correlation. This is an innovative mapping analysis that allows us to appreciate the marginal contribution of each voxel in the correlation of two maps, so as to better identify the voxels exerting more influence in the correlation. Specifically, to examine the regional differences between the four conditions, we applied the analysis leave-one-voxel-out so as to evaluate the voxels' contribution to the correlation (VCC) between the maps (Mancuso et al., 2019). Every pair of corresponding voxels $v_{i 1}$ and $v_{i 2}$ was removed from both maps, and after every removal, the Pearson's $r$ between the two maps was recalculated. The difference $r_{\mathrm{GLOBAL}}-R_{\mathrm{ViREMOVED}}$ between the original value of $r$ and the value obtained after removing the couple of voxels $v_{i 1}$ and $v_{i 2}$ was attributed to voxel $v_{i}$. This procedure was repeated for $v_{j}, v_{k}, \ldots$; then, 
Table 4. Significant ALE Results for the Suprasecond Motor Condition Meta-analysis

\begin{tabular}{|c|c|c|c|c|c|c|c|}
\hline \multirow[b]{2}{*}{ Cluster } & \multirow[b]{2}{*}{ Volume $\left(\mathrm{mm}^{3}\right)$} & \multirow[b]{2}{*}{ Label } & \multirow[b]{2}{*}{ Side } & \multirow{2}{*}{$\begin{array}{c}\text { Extrema } \\
\text { Value }\end{array}$} & \multicolumn{3}{|c|}{ Talairach } \\
\hline & & & & & $x$ & $Y$ & $Z$ \\
\hline \multirow[t]{7}{*}{1} & 19,008 & Superior frontal gyrus (BA 6) & $\mathrm{L}$ & 0.024 & -2 & 10 & 50 \\
\hline & & Medial frontal gyrus (BA 6) & $\mathrm{R}$ & 0.020 & 2 & 0 & 62 \\
\hline & & Cingulate gyrus (BA 32) & $\mathrm{R}$ & 0.018 & 6 & 20 & 36 \\
\hline & & Medial frontal gyrus (BA 6) & $\mathrm{L}$ & 0.011 & -10 & -22 & 46 \\
\hline & & Cingulate gyrus (BA 32) & $\mathrm{L}$ & 0.009 & -6 & 24 & 34 \\
\hline & & Medial frontal gyrus (BA 8) & $\mathrm{L}$ & 0.009 & -4 & 28 & 42 \\
\hline & & Paracentral lobule (BA 31) & $\mathrm{L}$ & 0.007 & -2 & -24 & 42 \\
\hline \multirow[t]{6}{*}{2} & 11,784 & Inferior frontal gyrus (BA 44) & $\mathrm{R}$ & 0.023 & 52 & 8 & 22 \\
\hline & & Insula (BA 13) & $\mathrm{R}$ & 0.020 & 42 & 12 & 6 \\
\hline & & Inferior frontal gyrus (BA 47) & $\mathrm{R}$ & 0.014 & 36 & 20 & -4 \\
\hline & & Claustrum & $\mathrm{R}$ & 0.010 & 30 & 14 & 6 \\
\hline & & Inferior frontal gyrus (BA 45) & $\mathrm{R}$ & 0.009 & 46 & 28 & 4 \\
\hline & & Superior temporal gyrus (BA 22) & $\mathrm{R}$ & 0.005 & 56 & 8 & 4 \\
\hline \multirow[t]{4}{*}{3} & 6824 & Mammillary body & $\mathrm{L}$ & 0.020 & -12 & -18 & 4 \\
\hline & & Lentiform nucleus (putamen) & & 0.013 & -18 & -6 & 12 \\
\hline & & Caudate body & $\mathrm{L}$ & 0.010 & -14 & 8 & 12 \\
\hline & & Lateral posterior nucleus (thalamu & $\mathrm{L}$ & 0.008 & -14 & -18 & 18 \\
\hline
\end{tabular}

\section{Contributors to Cluster 1}

3 foci from Dudukovic \& Wagner (2007)

3 foci from Stevens et al. (2007)

2 foci from Lewis \& Miall (2002)

2 foci from Larsson et al. (1996)

2 foci from Taniwaki et al. (2006)

2 foci from Rubia et al. (1998)

2 foci from Riecker et al. (2003)

2 foci from Macar et al. (2002)

1 focus from Wittmann et al. (2011) (Exp. A)

1 focus from Wittmann et al. (2011) (Exp. B)

1 focus from Wittmann et al. (2011) (Exp. C)

\section{Contributors to Cluster 2}

2 foci from Wittmann et al. (2010) (Exp. B)

2 foci from Coull et al. (2013) (Exp. A)

2 foci from Ackermann et al. (2001)

2 foci from Shergill et al. (2006)
1 focus from Wittmann et al. (2010b) (Exp. B)

1 focus from Wittmann et al. (2010b) (Exp. C)

1 focus from Murai \& Yotsumoto (2016)

1 focus from Lejeune et al. (1997)

1 focus from Jech et al. (2005)

1 focus from Coull et al. (2013) (Exp. A)

1 focus from Basso et al. (2003)

1 focus from Shergill et al. (2006)

1 focus from Riecker et al. (2006)

1 focus from Rekkas et al. (2005) (Exp. B)

1 focus from Macar et al. (2004)

1 focus from Wittmann et al. (2010b) (Exp. C)

1 focus from Murai \& Yotsumoto (2016)

1 focus from Lewis \& Miall (2002)

1 focus from Lejeune et al. (1997) 
Table 4. (continued)

\begin{tabular}{|c|c|c|c|c|c|c|}
\hline \multirow[b]{2}{*}{ Cluster } & \multirow[b]{2}{*}{ Volume $\left(\mathrm{mm}^{3}\right)$} & \multirow[b]{2}{*}{ Side } & \multirow{2}{*}{$\begin{array}{c}\text { Extrema } \\
\text { Value }\end{array}$} & \multicolumn{3}{|c|}{ Talairach } \\
\hline & & & & $x$ & $Y$ & $Z$ \\
\hline \multicolumn{2}{|c|}{1 focus from Wittmann et al. (2011) (Exp. A) } & \multicolumn{5}{|c|}{1 focus from Brunia et al. (2000) } \\
\hline \multicolumn{2}{|c|}{1 focus from Wittmann et al. (2011) (Exp. B) } & \multicolumn{5}{|c|}{1 focus from Basso et al. (2003) } \\
\hline \multicolumn{2}{|c|}{1 focus from Wittmann et al. (2011) (Exp. C) } & \multicolumn{5}{|c|}{1 focus from Taniwaki et al. (2006) } \\
\hline \multicolumn{2}{|c|}{1 focus from Wittmann et al. (2010b) (Exp. A) } & \multicolumn{5}{|c|}{1 focus from Stevens et al. (2007) } \\
\hline
\end{tabular}

\section{Contributors to Cluster 3}

3 foci from Riecker et al. (2006)

2 foci from Taniwaki et al. (2006)

2 foci from Riecker et al. (2003)

1 focus from Teki et al. (2016)
1 focus from Coull et al. (2013) (Exp. A)

1 focus from Ackermann et al. (2001)

1 focus from Stevens et al. (2007)

1 focus from Shergill et al. (2006)

For each cluster brain regions, cluster size $\left(\mathrm{mm}^{3}\right)$, side (hemisphere), ALE value, Talairach coordinates, and contributors to cluster (foci) were provided. $\mathrm{BA}=$ Brodmann's area; $\mathrm{L}=$ left; $\mathrm{R}=$ right.

the difference values were normalized, so as to obtain a voxel-wise estimation of the local contribution to the global correlation.

Negative VCC values denote the voxels whose removal produces an increase of the correlation, which means that they do not positively contribute to the global $r$. In contrast, positive VCC values denote the voxels contributing to a positive correlation between the two maps, whose removal determines an $r_{\text {GLOBAL }}$ decrease. The VCC can therefore provide us with the information about the regions that contribute to the similarity and the dissimilarity of the two maps.

\section{Hierarchical Clustering and Features of Relevance Estimation}

To better investigate the differences and commonalities between the various conditions, we applied a hierarchical clustering analysis using as entry elements the four obtained ALE maps. As a first step, the peak ALE values for each of the ROIs were calculated. Parcels were identified on the basis of the Talairach Atlas. The 113 ROIs that showed nonzero peak values at least in one of the four conditions were passed to subsequent analyses, performed using the Orange Canvas software. An $N \times$ $M$ matrix (4 conditions $\times 113$ ROIs) was used as input. Euclidean distances were computed between rows (i.e., between conditions), and hierarchical clustering was performed with the Ward's linkage method. We then used the information gain (IG) criterion (Alhaj, Siraj, Zainal, Elshoush, \& Elhaj, 2016) to identify the most informative brain regions capable of distinguishing between the four conditions. The IG is based on a measure of entropy and is commonly used in the feature-ranking procedures, so as to identify those features that are particularly relevant for the accurate implementation of a given task (e.g., to assign an item to the correct class among many).

\section{Reliability Analysis}

To investigate the reliability of the obtained results, we applied the "fail-safe" technique. This kind of approach, frequently used in classical meta-analyses, was first introduced by Rosenthal (1979), and recently, Acar et al. (2018) made it suitable for ALE meta-analyses. This method addresses the issue of the so-called "file-drawer effect," that is, the smaller chance for a study to show nonstatistically significant results to get published. This bias could lead to overestimated effects in meta-analyses because of the lack of contraevidences in the pool of considered studies. The procedure that we used, proposed by Acar, Seurinck, Eickhoff, and Moerkerke (2017; https://github. $\mathrm{com} /$ NeuroStat/GenerateNull), allows to introduce "noise experiments" to the meta-analysis pool. The injection of growing levels of noise raises the statistical threshold, allowing to test how many unpublished experiments should exist to nullify the obtained results. More in details, the Acar's algorithm observes the distributions of sample size and amount of reported foci per experiment in the pool of real experiments. These two parameters are pivotal for the implementation of the ALE method and the identification of the effect (Eickhoff et al., 2016). Noise experiments are then created randomly by combining values of sample size and amount of reported foci per experiment taken from the respective real distributions (Acar et al., 2017). The 
Table 5. Significant ALE Results for the Suprasecond Perceptual Condition Meta-analysis

\begin{tabular}{|c|c|c|c|c|c|c|c|}
\hline \multirow[b]{2}{*}{ Cluster } & \multirow[b]{2}{*}{ Volume $\left(\mathrm{mm}^{3}\right)$} & \multirow[b]{2}{*}{ Label } & \multirow[b]{2}{*}{ Side } & \multirow{2}{*}{$\begin{array}{c}\text { Extrema } \\
\text { Value }\end{array}$} & \multicolumn{3}{|c|}{ Talairach } \\
\hline & & & & & $x$ & $y$ & $z$ \\
\hline \multirow[t]{10}{*}{1} & 19,240 & Insula (BA 13) & $\mathrm{R}$ & 0.037 & 34 & 16 & 6 \\
\hline & & Inferior frontal gyrus (BA 44) & $\mathrm{R}$ & 0.025 & 50 & 10 & 20 \\
\hline & & Middle frontal gyrus (BA 9) & $\mathrm{R}$ & 0.016 & 36 & 32 & 32 \\
\hline & & Middle frontal gyrus (BA 10) & $\mathrm{R}$ & 0.011 & 32 & 44 & 2 \\
\hline & & Middle frontal gyrus (BA 46) & $\mathrm{R}$ & 0.010 & 44 & 40 & 26 \\
\hline & & Middle frontal gyrus (BA 6) & $\mathrm{R}$ & 0.009 & 30 & 16 & 50 \\
\hline & & Inferior frontal gyrus (BA 46) & $\mathrm{R}$ & 0.008 & 44 & 32 & 6 \\
\hline & & Middle frontal gyrus (BA 9) & $\mathrm{R}$ & 0.008 & 44 & 22 & 28 \\
\hline & & Precentral gyrus (BA 6) & $\mathrm{R}$ & 0.008 & 38 & 2 & 32 \\
\hline & & Inferior frontal gyrus (BA 45) & $\mathrm{R}$ & 0.007 & 60 & 24 & 12 \\
\hline \multirow[t]{11}{*}{2} & 16,848 & Medial frontal gyrus (BA 32) & $\mathrm{L}$ & 0.030 & -4 & 8 & 46 \\
\hline & & Medial frontal gyrus (BA 6) & $\mathrm{L}$ & 0.030 & -6 & 4 & 52 \\
\hline & & Superior frontal gyrus (BA 6) & $\mathrm{R}$ & 0.024 & 6 & 10 & 54 \\
\hline & & Cingulate gyrus (BA 32) & $\mathrm{R}$ & 0.013 & 12 & 28 & 26 \\
\hline & & Medial frontal gyrus (BA 8) & $\mathrm{R}$ & 0.012 & 2 & 22 & 46 \\
\hline & & Middle frontal gyrus (BA 6) & $\mathrm{R}$ & 0.012 & 30 & 4 & 62 \\
\hline & & Medial frontal gyrus (BA 9) & $\mathrm{R}$ & 0.011 & 2 & 34 & 30 \\
\hline & & Superior frontal gyrus (BA 9) & $\mathrm{R}$ & 0.011 & 22 & 40 & 30 \\
\hline & & Superior frontal gyrus (BA 6) & $\mathrm{R}$ & 0.010 & 22 & 6 & 60 \\
\hline & & Superior frontal gyrus (BA 6) & $\mathrm{R}$ & 0.009 & 16 & 16 & 54 \\
\hline & & Middle frontal gyrus (BA 6) & $\mathrm{R}$ & 0.008 & 30 & 2 & 52 \\
\hline \multirow[t]{7}{*}{3} & 16,016 & Claustrum & $\mathrm{L}$ & 0.033 & -34 & 14 & 4 \\
\hline & & Inferior frontal gyrus (BA 44) & $\mathrm{L}$ & 0.028 & -48 & 6 & 20 \\
\hline & & Lentiform nucleus (putamen) & $\mathrm{L}$ & 0.014 & -14 & 8 & -4 \\
\hline & & Lentiform nucleus (putamen) & $\mathrm{L}$ & 0.011 & -16 & 4 & 4 \\
\hline & & Middle frontal gyrus (BA 9) & $\mathrm{L}$ & 0.009 & -54 & 8 & 36 \\
\hline & & Middle frontal gyrus (BA 9) & $\mathrm{L}$ & 0.008 & -40 & 14 & 28 \\
\hline & & Precentral gyrus (BA 44) & $\mathrm{L}$ & 0.006 & -50 & 12 & 4 \\
\hline \multirow[t]{7}{*}{4} & 7368 & Inferior parietal lobule (BA 40) & $\mathrm{R}$ & 0.020 & 48 & -42 & 42 \\
\hline & & Inferior parietal lobule (BA 40) & $\mathrm{R}$ & 0.019 & 40 & -48 & 42 \\
\hline & & Insula (BA 13) & $\mathrm{R}$ & 0.009 & 52 & -34 & 20 \\
\hline & & Superior temporal gyrus (BA 13) & $\mathrm{R}$ & 0.009 & 52 & -42 & 22 \\
\hline & & Inferior parietal lobule (BA 40) & $\mathrm{R}$ & 0.009 & 54 & -32 & 46 \\
\hline & & Superior temporal gyrus (BA 41) & $\mathrm{R}$ & 0.009 & 54 & -28 & 10 \\
\hline & & Superior temporal gyrus (BA 22) & $\mathrm{R}$ & 0.005 & 54 & -38 & 10 \\
\hline
\end{tabular}


Table 5. (continued)

\begin{tabular}{|c|c|c|c|c|c|c|c|}
\hline \multirow[b]{2}{*}{ Cluster } & \multirow[b]{2}{*}{ Volume $\left(\mathrm{mm}^{3}\right)$} & \multirow[b]{2}{*}{ Label } & \multirow[b]{2}{*}{ Side } & \multirow{2}{*}{$\begin{array}{c}\text { Extrema } \\
\text { Value }\end{array}$} & \multicolumn{3}{|c|}{ Talairach } \\
\hline & & & & & $x$ & $y$ & $z$ \\
\hline
\end{tabular}

Contributors to Cluster 1

6 foci from Lewis \& Miall (2003b)

4 foci from Carvalho et al. (2016) (Exp. A)

4 foci from Smith et al. (2003)

3 foci from Pfeuty et al. (2015)

3 foci from Morillon et al. (2009)

3 foci from Coull et al. (2013) (Exp. B)

2 foci from Ustun et al. (2017)

2 foci from Jueptner et al. (1996)

2 foci from Coull et al. (2000)

\section{Contributors to Cluster 2}

3 foci from Coull et al. (2004)

3 foci from Carvalho et al. (2016)(Exp. A)

2 foci from Wiener et al. (2014)

2 foci from Morillon et al. (2009)

2 foci from Harrington et al. (2004)

2 foci from Coull et al. (2000)

2 foci from Smith et al. (2003)

2 foci from Pouthas et al. (2005)

1 focus from Ustun et al. (2017)

1 focus from Smith et al. (2011)

\section{Contributors to Cluster 3}

3 foci from Lewis \& Miall (2003b)

3 foci from Harrington et al. (2010)

3 foci from Carvalho et al. (2016) (Exp. A)

3 foci from Rao et al. (2001) (Exp. D)

2 foci from Ustun et al. (2017)

2 foci from Livesey et al. (2007)

2 foci from Coull et al. (2013) (Exp. B)

2 foci from Coull et al. (2000)

\section{Contributors to Cluster 4}

2 foci from Harrington et al. (2004)

2 foci from Carvalho et al. (2016) (Exp. B)

2 foci from Tsukamoto et al. (2006)
2 foci from Rao et al. (2001) (Exp. C)

1 focus from Wiener et al. (2014)

1 focus from Livesey et al. (2007)

1 focus from Harrington et al. (2004)

1 focus from Coull et al. (2004)

1 focus from Apaydin et al. (2018)

1 focus from Rao et al. (2001) (Exp. A)

1 focus from Rao et al. (2001) (Exp. B)

1 focus from Pouthas et al. (2005)

1 focus from Pfeuty et al. (2015)

1 focus from Lewis \& Miall (2003b)

1 focus from Herrmann et al. (2014)

1 focus from Harrington et al. (2010)

1 focus from Coull et al. (2013)(Exp. B)

1 focus from Apaydın et al. (2018)

1 focus from Rao et al. (2001) (Exp. A)

1 focus from Rao et al. (2001) (Exp. B)

1 focus from Rao et al. (2001) (Exp. C)

1 focus from Rao et al. (2001) (Exp. D)

2 foci from Tsukamoto et al. (2006)

2 foci from Rao et al. (2001) (Exp. C)

1 focus from Smith et al. (2011)

1 focus from Morillon et al. (2009)

1 focus from Herrmann et al. (2014)

1 focus from Harrington et al. (2004)

1 focus from Coull et al. (2004)

1 focus from Rao et al. (2001) (Exp. B)

1 focus from Lewis \& Miall (2003b)

1 focus from Coull et al. (2013) (Exp. B)

1 focus from Coull et al. (2004) 
Table 5. (continued)

\begin{tabular}{|c|c|c|c|c|c|c|c|}
\hline \multirow[b]{2}{*}{ Cluster } & \multirow[b]{2}{*}{ Volume $\left(\mathrm{mm}^{3}\right)$} & \multirow[b]{2}{*}{ Label } & \multirow[b]{2}{*}{ Side } & \multirow{2}{*}{$\begin{array}{c}\text { Extrema } \\
\text { Value }\end{array}$} & \multicolumn{3}{|c|}{ Talairach } \\
\hline & & & & & $x$ & $y$ & $z$ \\
\hline \multicolumn{3}{|c|}{1 focus from Ustun et al. (2017) } & \multicolumn{5}{|c|}{1 focus from Coull et al. (2000) } \\
\hline \multicolumn{3}{|c|}{1 focus from Smith et al. (2011) } & \multicolumn{5}{|c|}{1 focus from Apaydın et al. (2018) } \\
\hline
\end{tabular}

For each cluster brain regions, cluster size $\left(\mathrm{mm}^{3}\right)$, side (hemisphere), ALE value, Talairach coordinates, and contributors to cluster (foci) were provided. $\mathrm{BA}=$ Brodmann's area; $\mathrm{L}=$ left; $\mathrm{R}=$ right.

final step is the repetition of the ALE analysis using as input the real pool injected with variable amount of noise experiments.

In this study, the range of injected noise was between $\frac{k}{4}$ and $3 k$, where $k$ is the number of real experiments. In each repetition, we added the $\frac{k}{4}$ of noise (i.e., 12 ALE maps). Finally, we created a map showing the residual real contribution of each brain area after an increasing injection of noise. Because the sample size and the amount of reported foci per experiment are specific for each pool used for a meta-analysis, we repeated the fail-safe procedure for each of the four conditions investigated in this study.

\section{RESULTS}

\section{The Patterns of Brain Areas Involved in Time Perception}

The ALE analysis of the subsecond motor condition shows activations mainly localized in medial and superior frontal gyri, left precentral gyrus, right cingulate gyrus, left superior temporal gyrus, left inferior parietal lobule, insula, right claustrum, thalamus, putamen, right caudate body, substantia nigra, and cerebellum (Figure 2 and Table 2).

The ALE analysis of the subsecond perceptual condition shows activations mainly localized in the left claustrum, left middle, medial and inferior frontal gyri, left precentral gyrus, left putamen, insula, right caudate body, right superior temporal gyrus, right globus pallidus, left cingulate gyrus, and right superior frontal gyrus (Figure 2 and Table 3).
The ALE analysis of the suprasecond motor condition shows activations mainly localized in left superior frontal gyrus, medial frontal gyrus, cingulate gyrus, right inferior frontal gyrus, right insula, right claustrum, right superior temporal gyrus, left mammillary body, left putamen, left caudate body, and left thalamus (Figure 2 and Table 4).

The ALE analysis of the suprasecond perceptual condition shows activations mainly localized in right insula, right middle and inferior frontal gyri, precentral gyrus, left medial frontal gyrus, right superior frontal gyrus, right cingulate gyrus, right medial frontal gyrus, left claustrum, left inferior frontal gyrus, left putamen, left middle frontal gyrus, right inferior parietal lobule, and right superior temporal gyrus (Figure 2 and Table 5).

\section{Correlation between Maps and Voxels' Contribution to Correlation Analysis}

With regard to the correlation analyses, the highest value $(r=.51)$ was found between subperceptual and supraperceptual conditions (Table 6). The correlation between supramotor versus supraperceptual was $r=.38$, followed by submotor versus supramotor $(r=.36)$ and submotor versus subperceptual $(r=.23)$. In light of this, the submotor condition is more correlated with the supramotor than the subperceptual. The subperceptual condition is more correlated with the supraperceptual than the submotor. The supramotor condition is more correlated with the supraperceptual than the submotor. Finally, the supraperceptual condition is more correlated with the subperceptual than the supramotor.

Table 6. Correlation Values (Pearson's $r$ ) between Each Couple of Conditions

\begin{tabular}{|c|c|c|c|c|}
\hline & Subsecond motor & Subsecond perceptual & Suprasecond motor & Suprasecond perceptual \\
\hline Subsecond motor & & 0.23 & 0.36 & 0.18 \\
\hline Subsecond perceptual & & & 0.37 & 0.51 \\
\hline Suprasecond motor & & & & 0.38 \\
\hline
\end{tabular}




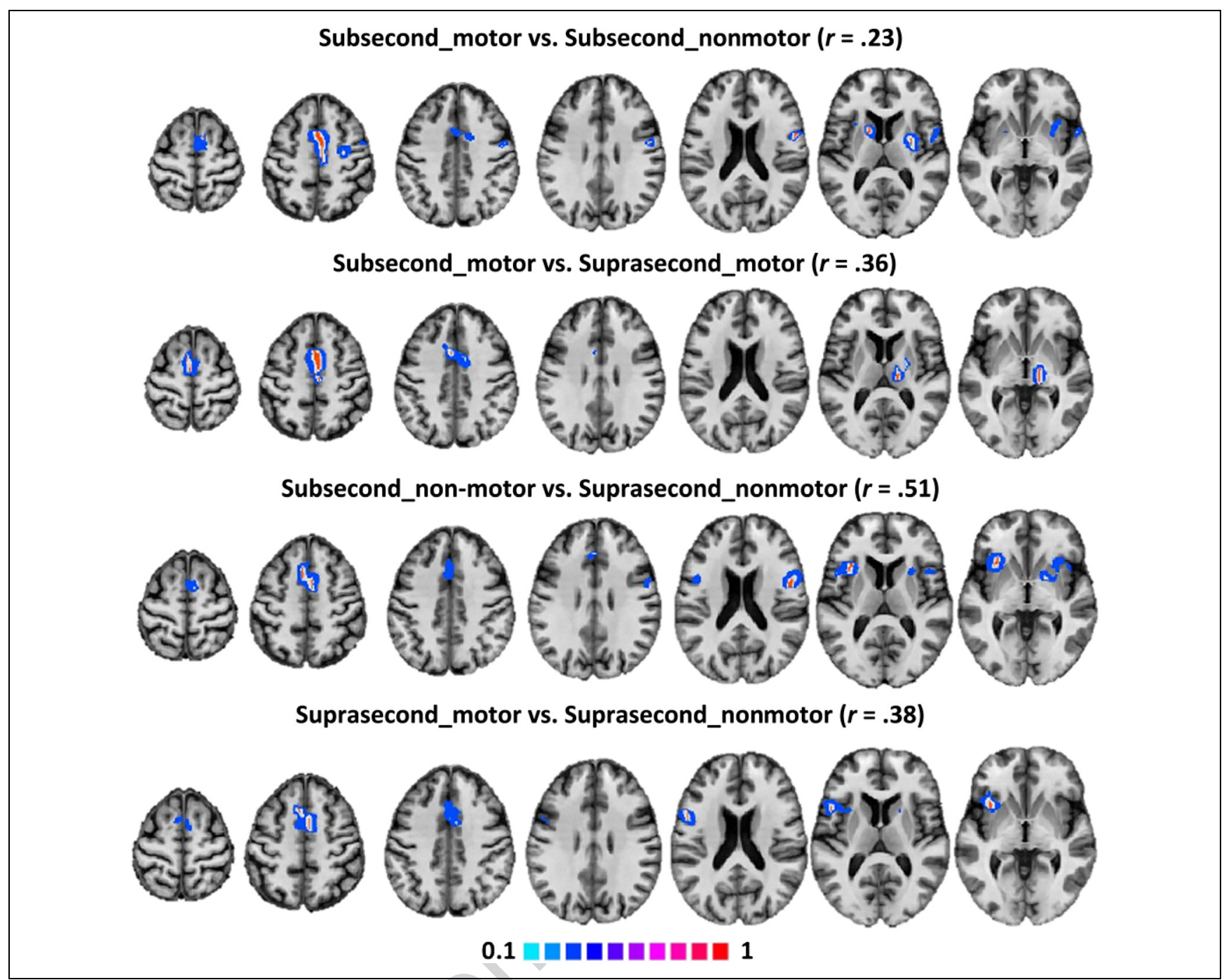

Figure 3. Results of the contribution leave-one-voxel-out to the correlation analyses. Colors from light blue to red represent increasing contribution to the overall correlation between the two conditions (values were normalized between 0 and 1). Values of $r$ are the results of the correlation before the leave-one-out procedure. Slices on the axial plane are shown in radiological convention (i.e., right is left, left is right).

The VCC analysis produced the following results. The voxels driving the correlation between the subsecond motor and subsecond perceptual conditions are mainly localized in the left superior and middle frontal gyrus, left superior insula, right caudate body, left precentral gyrus, and right lateral globus pallidus (Figure 3).

The voxels driving the correlation between the subsecond motor and suprasecond motor conditions are mainly localized in the bilateral medial frontal gyrus, the left ventral posterior lateral nucleus of the thalamus, and the left putamen (Figure 3).

The voxels driving the correlation between the subsecond perceptual and the suprasecond perceptual conditions are mainly localized in the right superior and medial frontal gyrus, bilateral anterior insula, left inferior frontal gyrus, and left putamen (Figure 3).

The voxels driving the correlation between the suprasecond motor and the suprasecond perceptual conditions are mainly localized in the right superior, medial and inferior frontal gyri, and left putamen (Figure 3).

\section{Hierarchical Clustering and Features of Relevance Estimation}

The dendrogram obtained through the hierarchical clustering shows the highest similarity between the two perceptual conditions (i.e., sub- and suprasecond). The suprasecond motor condition results to be more similar to the perceptual cluster than to the subsecond motor condition (Figure 4). This configuration is coherent with the correlation results, because the highest value was found between the two perceptual conditions. The suprasecond motor condition shows a slightly higher correlation with the suprasecond perceptual condition rather than with the subsecond motor one. The IG identifies the left $\mathrm{BA} 31$, left and right putamen, right $\mathrm{BA} 6$, right 
Figure 4. Results of the hierarchical clustering. Dendrogram was built using the Ward's linkage. The nodes' values represent dissimilarity.

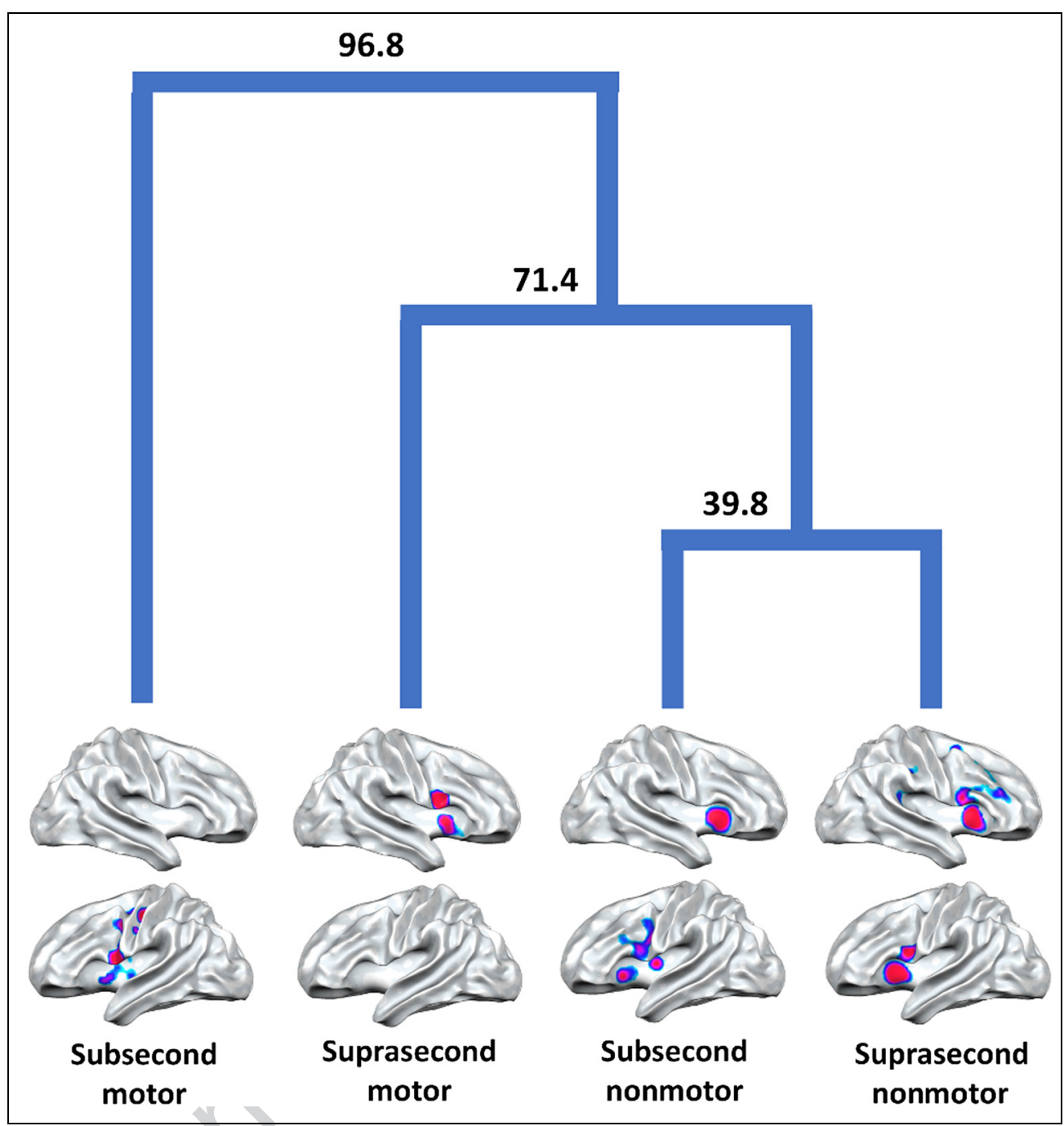

BA 32, left BA 45, left BA 8, right ventral anterior nucleus of the thalamus, left lateral and medial globus pallidus, right $\mathrm{BA} 44$, left posterior and anterior BA 13, left BA 22, left BA 47, left caudate body, left BA 43, left BA 4, and right BA 24 as the most informative areas for discriminating between conditions. Of note, none of these brain regions was active in only one of the four conditions.

The overlap of the patterns of activations obtained with the ALE maps associated with the four conditions identifies the SMA as the area in which conditions show the greatest overlap (100\%; Figure 5).

As the SMA appears to be the area with the greatest overlap between conditions, we have examined how its activation can vary in relation to sub- and suprasecond tasks, as well as to motor and nonmotor tasks (Figure 6).

\section{Reliability}

The fail-safe analysis shows a good resistance of the results (Figure 7). Most of the activation blobs, across the four conditions, survive after the $50 \%$ level of noise is injected. Of note, the SMA is still present even after the

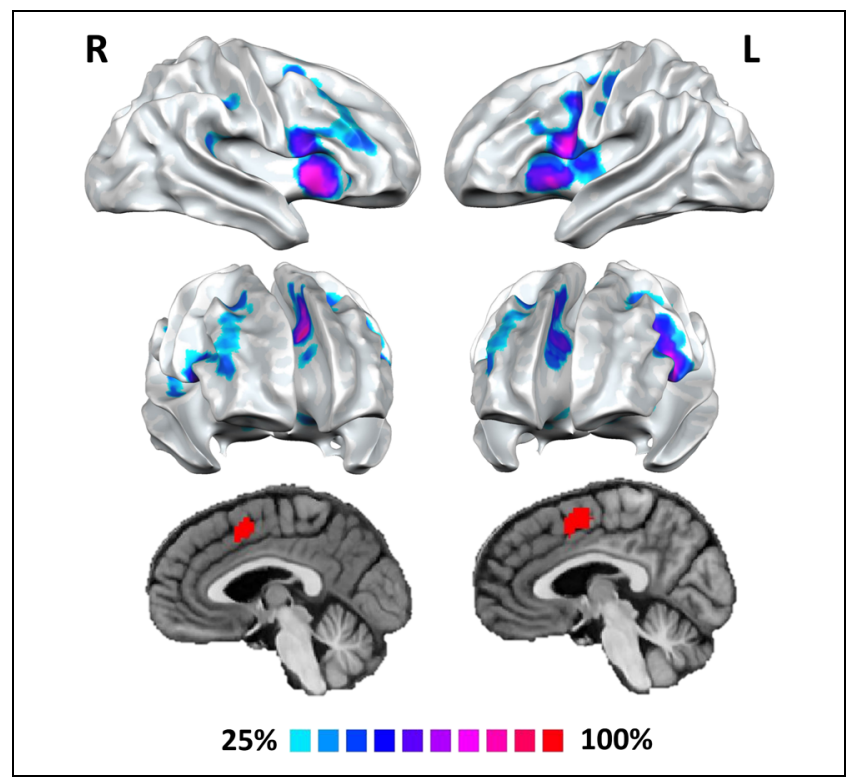

Figure 5. Overlap between the ALE maps associated to each of the four conditions. Colors from light blue to red represent increasing overlap values. Voxels showing value equal to $100 \%$ were found to be active through all the four conditions. Slices on the axial plane are shown in radiological convention (i.e., right is left, left is right). 
Figure 6. Sagittal and axial sections of the ALE in all the four conditions in which the different activation profile of the SMA can be appreciated. Colors from light blue to red represent increasing ALE values. Slices on the axial plane are shown in radiological convention (i.e., right is left, left is right).

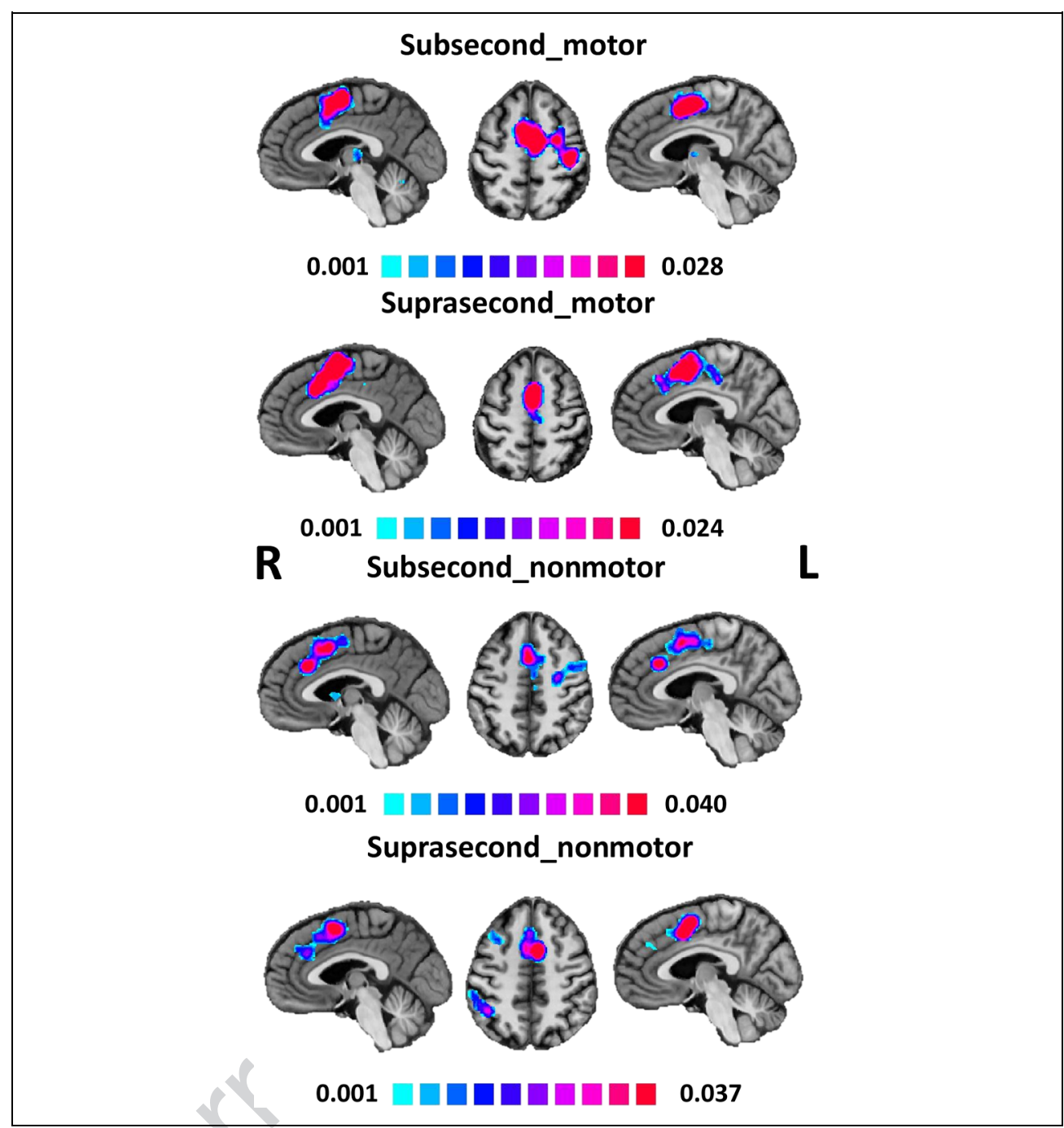

injection of the $300 \%$ of noise in three of the four conditions (the exception is the subsecond perceptual). Subcortical regions show a good resistance, especially with regard to the subsecond conditions (motor and nonmotor), where they survive above the $50 \%$ of the noise level. A similar trend is observed for the insula as well.

Overall, the fail-safe analysis about the robustness of our results is reassuring, as most of them have maintained significance after noise injections of $50 \%$ and the SMA even after an injection of $300 \%$ of noise.

\section{DISCUSSION}

Our meta-analytical investigation, which used a new corrected version for the ALE and an innovative fail-safe technique, mostly confirms the findings of the previous meta-analyses. However, patterns of activation related to subsecond and suprasecond tasks are much more similar than previously thought. Both subsecond and suprasecond tasks recruit cortical and subcortical areas, but subcortical areas contribute more to the pattern associated with subsecond tasks than to the pattern associated with suprasecond tasks, which instead receives more contributions from cortical activations. This is in line with the idea that distinct timing mechanisms may operate at different timescales (Buhusi \& Meck, 2005), as well as to the idea that intervals below the 1-sec range are supposed to be more dependent on sensory and automatic processes, whereas the detection and estimation of intervals longer than $1 \mathrm{sec}$ are thought to rely more on cognitive functions (Lewis \& Miall, 2003a; Rammsayer, 1999). Several cognitive processes are in fact likely to contribute to the perception/construction of time (Meck, 2005; Pouthas \& Perbal, 2004). For instance, the degree of attention can modulate and influence the estimate of time (Coull, Vidal, Nazarian, \& Macar, 2004; Nobre \& O'Reilly, 2004). Also, working memory and executive processes come into play in time judgments (Radua, Del Pozo, Gomez, Guillen-Grima, \& Ortuño, 2014). In tasks when the participant is required to compare the different durations of two stimuli, the duration of the first stimulus must be held in the STM storage so as to decide which stimulus is longer (Livesey, Wall, \& Smith, 2007). 


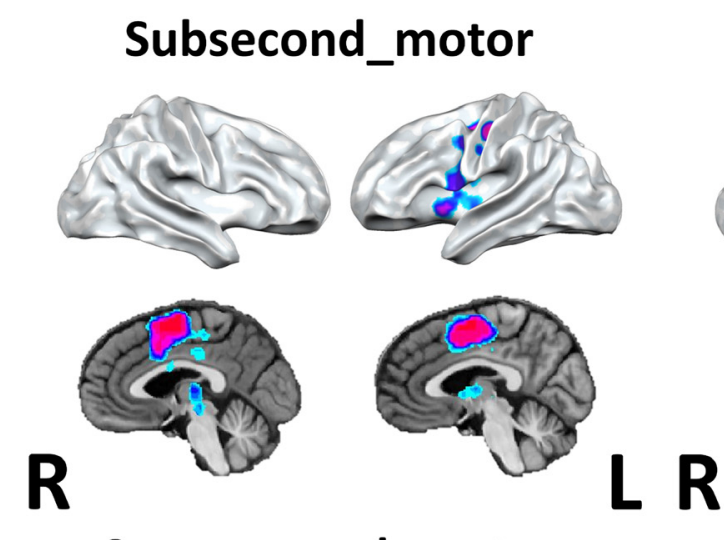

Subsecond_nonmotor

Suprasecond_motor

Suprasecond_nonmotor
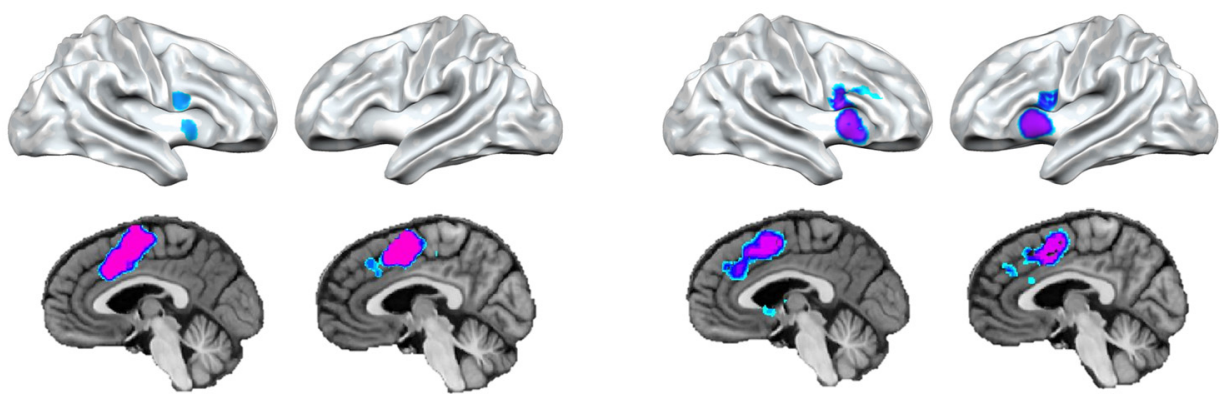

$10 \% \square \square+\square \square \square \square \square \square-100 \%$

Figure 7. Results of the fail-safe analyses for the four conditions. Colors from light blue to red represent increasing resistance to injected noise. Slices on the axial plane are shown in radiological convention (i.e., right is left, left is right).

Motor and perceptual conditions have similar profiles, but the pattern associated with the motor condition is more extended in subcortical and parietal sites than the pattern associated with the perceptual condition. The recruitment of parietal sites is probably due to the wellknown functions of these cortical areas in the elaboration of the where and how of spatial vision (Oliveri et al., 2009; Alexander, Cowey, \& Walsh, 2005), which are fundamental for building spatiotemporal representations and then voluntarily orienting the motor response (but see further on for a more detailed discussion of this point).

Overall, our analysis provides evidence that the patterns associated with the four conditions are characterized by many common activations in cerebral sites; in particular, they share activations in the following regions: the insula, superior, medial and inferior frontal gyri, precentral gyrus, cingulate gyrus, superior temporal gyrus, claustrum, putamen, and caudate body. This various set of brain areas highlights the importance of the interplay between cortical and subcortical components, especially of frontal insular areas and the striatum, in performing tasks related to time (Wittmann, 2013; Wittmann \& van Wassenhove, 2009).

The insular cortex is thought to integrate different signals originating from the outside world and within the organism, a function that is fundamental for building an interoceptive feeling of the state of the body (Critchley, Wiens, Rotshtein, Ohman, \& Dolan, 2004; Craig, 2002). This integration would rely on an accumulation mechanism of physiological changes in the posterior insula (Wittmann, Simmons, Aron, \& Paulus, 2010) and then proceed in sequential representations of homeostatic states in a posteriorto-anterior progression (Craig, 2009b; Singer, Critchley, $\&$ Preuschoff, 2009). This would result in a unified metarepresentation capable of forming our internal feelings moment after moment, the development of which would be at the basis of our inner experience of duration (Craig, 2009a).

Undoubtedly, the great involvement of the frontal cortex (superior, medial, middle, and inferior gyri) in all the four conditions points, as already suggested, to the relevance of higher order cognitive processes, such as attention and working memory, to perform time estimation tasks, which in terms of cognitive resources are quite demanding (Radua et al., 2014). In particular, the SMA (which corresponds to parts of the superior and medial frontal gyri) has been variously associated with both sensory and sensorimotor processing of temporal intervals, with the rostral SMA (or pre-SMA) more engaged in sensory, nonsequential and suprasecond temporal processing, and the caudal SMA (or SMA-proper) more engaged in sensorimotor, sequential and subsecond temporal processing (Schwartze et al., 2012). Recently a study by Protopapa 
et al. (2019) has suggested that the entire SMA may codify chronomaps in a progressive way, with the spatial progression following a typical rostro-caudal direction, with anterior activations (those of pre-SMA) more associated with the processing of shorter time periods, and posterior activations (those of SMA-proper) more associated with the processing of longer time periods. As can be seen by the SMA activations illustrated by Figure 7, our results further support the rostrocaudal gradient proposed by Schwartze et al. (2012), who associate the activity of the pre-SMA and SMA-proper not only to the elaboration of different duration of time intervals but also to different tasks. Our results provide evidence for this view, as the gradient in the activation of the SMA appears to be associated not only with the length of the interval but also with the nature of the tasks, as caudal activations seem to be more related to the motor condition whereas rostral activations seem to be more related to the nonmotor (or perceptual) condition. This finding is also in line with what was reported by Wiener et al. (2011).

The activation of the precentral gyrus, especially the left one, may be related to information rehearsal (Rao et al., 2001) and directed attention (Tzourio et al., 1997). As already observed by Wiener et al. (2010), this area would be involved in maintaining task-directed attention. Furthermore, parts of the precentral gyrus may differ with respect to functional specificity: The equivalent location of the hand area on the motor homunculus may be associated with subsecond tasks, whereas the equivalent location of the mouth site may be associated with suprasecond tasks. In particular, subsecond tasks might engage the precentral gyrus in real (or imagined) timed hand movements (Oullier, Jantzen, Steinberg, \& Kelso, 2005), whereas suprasecond tasks might engage this area in vocal (or subvocal) counting strategies (Hinton, Harrington, Binder, Durgerian, \& Rao, 2004).

The cingulate cortex, in turn, lies at the crossroads of emotion, sensation, and action. In particular, the ACC is largely connected with the anterior insula, and both areas are essential parts of the salience network (Seeley et al., 2007); therefore, it might support the implementation of attentional and executive control in temporal tasks.

As already seen, the striatum has been repeatedly related to time perception. In virtue of its many connections with cortical regions, this subcortical structure would play the role of an "internal clock" capable of integrating the oscillatory cortical activity. More specifically, the striatal beat frequency (SBF) model proposed by Matell and Meck $(2000,2004)$ suggests that synchronized frequencies of large cortical areas are the neural inputs constituting the time code for constructing temporal representations. According to the SBF model, the dopaminergic system gives a burst of dopamine to the striatum, thus providing the onset signal for timing a certain stimulus (Meck et al., 2008). The spiny neurons of the striatum would then detect the concurrent activity of specific beat patterns of cortical fluctuations. Our results provide further support for the SBF model. Contributions of the striatum are in fact present in all the activation patterns associated with the four conditions of temporal processing.

Activity in the superior temporal gyrus has been related to temporal discrimination and reproduction of auditory stimuli (Jahanshahi, Jones, Dirnberger, \& Frith, 2006; Rao et al., 2001; Sakai et al., 1999), as well as to the generation of rhythms learned through the auditory modality (Bengtsson, Ehrsson, Forssberg, \& Ullen, 2005; Lewis, Wing, Pope, Praamstra, \& Miall, 2004; Jancke, Loose, Lutz, Specht, \& Shah, 2000; Rao et al., 1997). This region, therefore, plays an important role in the timing of auditory stimuli (Bueti, van Dongen, \& Walsh, 2008). However, it has also reported activity in this area in the temporal discrimination of visual stimuli (Coull et al., 2004; Ferrandez et al., 2003). As suggested by the authors of these studies and given that the evaluation of temporal intervals is more difficult for visual than for auditory stimuli (Westheimer, 1999), activation of the superior temporal gyrus may support an auditory imagery strategy or subvocalization, which could help discriminate visual durations.

An interesting finding that has not been reported by previous meta-analyses about time perception, with the exception of Wiener et al. (2012), is the contribution of the claustrum. Activations of this region, whose exact function still remains an unresolved mystery, are present in every pattern associated with the four experimental conditions. The claustrum has many distributed connections to practically all the cortical areas (frontal, premotor, motor, ventral temporal, entorhinal, ventral anterior cingulate, visual, somatosensory, and olfactory), as well as to important subcortical structures (lateral amygdala, putamen, caudate nucleus, and globus pallidus; Baizer, Sherwood, Noonan, \& Hof, 2014; Mathur, 2014; Torgerson \& Van Horn, 2014). In virtue of these extensive projections, Crick and Koch (2005) proposed that the claustrum might integrate different sensations into a unitary conscious representation. This proposal has been further developed by Smythies, Edelstein, and Ramachandran (2012, 2014), who put forward a detailed hypothesis of how the claustrum might bring about this temporal integration. According to this hypothesis, the claustrum may serve as a "synchrony detector," thus capable of elaborating higher order temporal patterns to coordinate and bind information coming from all over across the brain. Of note, our analysis shows that the claustrum has significant activations in the perceptual conditions. This provides further evidence that the claustrum might play an important role in constructing the temporal infrastructure of consciousness or, as we prefer to call it, the property of consciousness of being a "temporal glue" capable of binding different percepts in a coherent conscious scene. If the claustrum is to be seen as having this role, there seems to be in the brain two regions that are supposed to be "synchrony detectors" for temporal patterns (i.e., the claustrum and the SBF). We do not think that this situation is contradictory, but 
rather, we think that data can be reconciled if we consider, as we have noted, that the claustrum is much more activated in perceptual (and cognitive) tasks whereas the striatum appears to be more involved in motor tasks. The two "synchrony detectors" may, therefore, work in synergy with the prevalence of either one or the other according to the nature and demands of the current task.

Another difference with previous meta-analyses is the absence of the cerebellum in three of the activation patterns associated with the temporal conditions. In past studies, the cerebellum has been related to the elaboration of temporal information (Mangels, Ivry, \& Shimizu, 1998; Jueptner et al., 1995; Ivry, 1993; Ivry \& Keele, 1989). More recently, the cerebellum has been linked to the processing of duration-based rather than beat-based intervals (Cope, Grube, Singh, Burn, \& Griffiths, 2014; Grube, Cooper, Chinnery, \& Griffiths, 2010). Furthermore, it has been proposed that it could play a role in the neural network capable of timing short intervals, as it has been found to be more involved in subsecond rather than in suprasecond tasks (Fierro et al., 2007; Koch et al., 2007; Lee et al., 2007). The label-based meta-analysis carried out by Lewis and Miall (2003a) has confirmed the involvement of cerebellum in time processing. In particular, the authors found the activation of the right cerebellum in subsecond tasks and the activation of the left cerebellum in suprasecond tasks. The review of Penney and Vaitilingam (2008), which also maintained the division of experiments between subsecond and suprasecond categories, found that the cerebellum was the most frequently activated structure in subsecond timing tasks. But as pointed out by Wiener et al. (2010), this review included activations that were subtracted both from control tasks and from the rest condition, thus making it difficult to distinguish between activations related to timing procedures or to other cognitive processes. Finally, Wiener et al. (2010) reported the cerebellum to be active in the subsecond category only. Our results confirm in part the findings of these meta-analyses. As already said, activations of the cerebellum are totally absent in three of the four conditions taken into examination here, save for the subsecond motor. A possible explanation for this result is that the other meta-analyses have probably used less restrictive statistical thresholds. In fact the cerebellum was clearly present only in our unthresholded ALE maps, which suggests that beyond a certain statistical threshold its activation becomes nonsignificant. On the other hand, this result may be more likely due to the large sample of studies used in this meta-analysis. Although all the selected studies conducted whole-brain analyses, not all of them reported activity in the cerebellum. More specifically, the activation foci of the cerebellum found by experiments were as follows: For subsecond motor, 19 (79\%) reported activation foci, and 5 (21\%) did not. For subsecond perceptual, 13 (52\%) reported activation foci, and 12 (48\%) did not. For suprasecond motor, 13 (38\%) reported activation foci, and 21 (62\%) did not. Finally, for suprasecond perceptual, 9 (35\%) reported activation foci, and 17 (65\%) did not. In light of these data, the cerebellar activity does not seem to be so relevant as to contribute effectively to the processing of timing tasks that do not pertain to the subsecond motor condition.

Another result of this meta-analysis that contrasts with previous findings is the activation of the parietal cortex. Lewis and Miall (2003a) found this region activated in suprasecond tasks, whereas Wiener et al. (2010) reported activations of this area (especially the inferior parietal lobe) in subsecond motor, subsecond perceptual and suprasecond motor tasks, but not in suprasecond perceptual tasks. Contrary to Lewis and Miall (2003a) and in partial agreement with Wiener et al. (2010), we found activations of the parietal cortex in subsecond and motor tasks only. The activations were mainly restricted to the inferior parietal lobule, with the exception of the postcentral gyrus. These findings support the idea that the parietal cortex, in particular the inferior parietal lobule, could play a role in estimating time; however, what it does exactly remains unclear. Leon and Shadlen (2003) found activation of the posterior parietal cortex (i.e., lateral inferior lobule) of macaques in subsecond tasks. On the other hand, Oliveri et al. (2009) applied TMS on the parietal cortex to test healthy participants and patients with right brain damage during a task requiring setting the midpoint of a time suprasecond interval. The authors found that inhibition of the right posterior parietal cortex induces a directional bias in the time bisection task. In turn, Vicario, Martino, and Koch (2013) applied to two groups of healthy adults a transcranial direct current stimulation (anodal, cathodal, or sham) over the left and right posterior parietal cortices and compared the performance of the groups in suprasecond time reproduction tasks. Results showed that, when cathodal stimulation was applied to the right posterior parietal cortex, the temporal accuracy was affected and led participants to overestimate time intervals, whereas when it was applied to the left posterior parietal cortex, the variability in reproducing temporal intervals was reduced. The authors reported no effect for the anodal stimulation. As functions of the parietal cortex are intimately associated with attentional direction and spatial processing, it is extremely difficult to separate these components in timing tasks. The hope is that in the future better devised experimental protocols could help distinguish the contributions of parietal regions to time processing on the one hand and to spatial and attentional processes on the other.

The correlation analysis between the four experimental categories reveals that the strongest couple is subsecond perceptual and suprasecond perceptual, followed by suprasecond motor and suprasecond perceptual, subsecond motor and suprasecond motor, and subsecond motor and subsecond perceptual. In other words, the patterns of activations associated with the perceptual conditions exhibit a greater similarity than the patterns 
of activations associated with the motor conditions. These results further suggest that the frontal components, which are predominant in the perceptual conditions, are more specific for the nature of the task rather than for time duration. Furthermore, the subdivision between motor and perceptual processing may be due to the fact that these two conditions rely on different functional patterns of frontal sites, which in part have common activations, as it is also suggested by the VCC analysis, which shows that a variety of frontal voxels contribute to the correlations.

The hierarchical clustering analysis confirms the correlation results, as it shows that the highest similarity is between sub- and suprasecond perceptual conditions. Also the result that the suprasecond motor condition is slightly more similar with the suprasecond perceptual condition is in line with the correlation analysis (according to which the more correlated second couple is suprasecond motor and suprasecond perceptual). This may indicate that the similarity is mostly guided by the frontal components, which are predominant in the suprasecond perceptual condition.

The IG analysis gives us the areas in which we can observe the major differences in peak activation between the four conditions. Interestingly, 13 of 19 areas are localized in the left hemisphere, and among those, most are frontal regions, which suggests that part of the left frontal pattern may have the role of discriminating tasks in each condition.

Finally, different from the meta-analysis of Wiener et al. (2010), which found only two areas (i.e., the SMA and the inferior frontal gyrus) with significant activations in a conjunction analysis of all the timing conditions, our overlap of the patterns of activations obtained with the ALE maps associated with the four conditions reveals just the SMA as the area of $100 \%$ of overlap. This provides further evidence for the pivotal role of the SMA in estimating time intervals, independent of the condition.

The SMA, along subcortical structures and the insula, are the areas that survive better the fail-safe procedure. These brain regions, therefore, might be considered as core network nodes for time perception, thus suggesting that our sense of time could emerge from the complex interaction of higher order and automatic cognitive processes.

\section{Limitations and Future Directions}

A concern with the ALE approach, which is, however, one of the most applied methods in coordinate-based metaanalyses, is that results might be driven by few experiments, thus representing a particular case among the ones selected for the meta-analysis rather that an overall descriptive view. Still, it should be observed that a minimum amount of 20 experiments is generally considered to be sufficient to avoid this issue (Eickhoff et al., 2016), so that analyses on large databases, as the one carried out here, should not be biased. Also, our sample is very well balanced, as we performed our analysis on a comparable sample size for each condition. However, although we were able to identify a considerable number of studies, others could have been omitted.

Future studies are needed to unravel the brain mechanisms at the root of our perception/construction of time. We need especially better protocols so as to distinguish in more details the roles played by cerebral structures in temporal processing. For instance, the activity of primary sensorimotor areas might be merely due to real or imaginary counting strategies. It is therefore fundamental to understand in what degree the areas responsible for sensory perception and motor ideation and execution contribute to the estimation of time per se. Finally, whether or not the cerebellum should be considered an essential brain structure in time processing is still a moot point, as it does not show concordance across studies. Our results suggest that its involvement is associated with the subsecond motor but not with the other conditions. Further analyses are needed to address this interesting issue.

\section{Conclusion}

The variability of time perception models may be accounted for by the peculiar nature of temporal phenomena, which are not directly related to objects in the outside world and for which we do not possess a dedicated sensory organ. This meta-analysis provides evidence for the existence of specific patterns of activations that can be associated with the perception and/or estimation of time in subsecond and suprasecond motor and nonmotor tasks. These patterns, however, are not radically different but have in part similar profiles of activations, as they share the contributions of common brain areas. Motor and subsecond tasks recruit slightly more contributions of subcortical structures than perceptual and suprasecond tasks, which in turn show patterns of activations more associated with cortical areas. All the conditions, however, exhibit a strong component of frontal areas and, in particular, a robust activation of the SMA. Rostral and caudal parts of this area play a pivotal role in discriminating not only different time intervals but also in relation to the nature of the task conditions. The SMA, in addition with the striatum (especially the putamen) and the claustrum, might constitute a core circuit in the different networks engaged when the sense of time is constructed by the brain.

\section{Acknowledgments}

This study was supported by the Fondazione Carlo Molo and Fondazione Sanpaolo (F. Cauda, PI), Turin. A. N. conceived the experiment, implemented data collection, drafted, and revised the article. J. M. analyzed the data, drafted, and revised the article. L. D. implemented data collection, drafted, and revised the article. S. D. revised the article. T. C. devised the tools of analysis, supervised the analysis of data, drafted, and revised the article. F. C. supervised the analysis of data, drafted, and revised the article. 
Reprint requests should be sent to Tommaso Costa, Department of Psychology, Via Po 14, 10123 Turin, Italy, or via e-mail: tommaso. costa@unito.it.

\section{REFERENCES}

Acar, F., Seurinck, R., Eickhoff, S. B., \& Moerkerke, B. (2018). Assessing robustness against potential publication bias in activation likelihood estimation (ALE) meta-analyses for fMRI. PLOS ONE, 13, e0208177.

Ackermann, H., Riecker, A., Mathiak, K., Erb, M., Grodd, W., \& Wildgruber, D. (2001). Rate-dependent activation of a prefrontal-insular-cerebellar network during passive listening to trains of click stimuli: An fMRI study. NeuroReport, 12, 4087-4092.

Alexander, I., Cowey, A., \& Walsh, V. (2005). The right parietal cortex and time perception: Back to Critchley and the Zeitraffer phenomenon. Cognitive Neuropsychology, 22, 306-315.

Alhaj, T. A., Siraj, M. M., Zainal, A., Elshoush, H. T., \& Elhaj, F. (2016). Feature selection using information gain for improved structural-based alert correlation. PLOS ONE, 11 e0166017.

Apaydın, N., Üstün, S., Kale, E. H., Çelikağ, İ., Özgüven, H. D., Baskak, B., et al. (2018). Neural mechanisms underlying time perception and reward anticipation. Frontiers in Human Neuroscience, 12, 115

Aso, K., Hanakawa, T., Aso, T., \& Fukuyama, H. (2010). Cerebrocerebellar interactions underlying temporal information processing. Journal of Cognitive Neuroscience, 22, 2913-2925.

Baizer, J. S., Sherwood, C. C., Noonan, M., \& Hof, P. R. (2014). Comparative organization of the claustrum: What does structure tell us about function? Frontiers in Systems Neuroscience, 8, 117.

Basso, G., Nichelli, P., Wharton, C. M., Peterson, M., \& Grafman J. (2003). Distributed neural systems for temporal production: A functional MRI study. Brain Research Bulleting, 59, 405-411.

Battelli, L., Pascual-Leone, A., \& Cavanagh, P. (2007). The 'when' pathway of the right parietal lobe. Trends in Cognitive Sciences, 11, 204-210.

Bengtsson, S. L., Ehrsson, H. H., Forssberg, H., \& Ullen, F. (2005). Effector-independent voluntary timing: Behavioural and neuroimaging evidence. European Journal of Neuroscience, 22, 3255-3265.

Bengtsson, S. L., Ullén, F., Ehrsson, H. H., Hashimoto, T., Kito, T., Naito, E., et al. (2009). Listening to rhythms activates motor and premotor cortices. Cortex, 45, 62-71.

Berns, G. S., Cohen, J. D., \& Mintun, M. A. (1997). Brain regions responsive to novelty in the absence of awareness. Science, 276, 1272-1275.

Beudel, M., Renken, R., Leenders, K. L., \& de Jong, B. M. (2009). Cerebral representations of space and time. Neuroimage, 44, 1032-1040.

Botzung, A., Denkova, E., \& Manning, L. (2008). Experiencing past and future personal events: Functional neuroimaging evidence on the neural bases of mental time travel. Brain and Cognition, 66, 202-212.

Brunia, C. H. M., de Jong, B. M., van den Berg-Lenssen, M. M. C., \& Paans, A. M. J. (2000). Visual feedback about time estimation is related to a right hemisphere activation measured by PET. Experimental Brain Research, 130, 328-337.

Bueti, D., van Dongen, E. V., \& Walsh, V. (2008). The role of superior temporal cortex in auditory timing. PLOS ONE, 3 , e2481.
Bueti, D., Walsh, V., Frith, C., \& Rees, G. (2008). Different brain circuits underlie motor and perceptual representations of temporal intervals. Journal of Cogntive Neuroscience, 20 , 204-214.

Buhusi, C. V., \& Meck, W. H. (2005). What makes us tick? Functional and neural mechanisms of interval timing. Nature Reviews Neuroscience, 6, 755-765.

Buonomano, D. V., \& Maass, W. (2009). State-dependent computations: Spatiotemporal processing in cortical networks. Nature Reviews Neuroscience, 10, 113-125.

Carvalho, F. M., Chaim, K. T., Sanchez, T. A., \& de Araujo, D. B. (2016). Time-perception network and default mode network are associated with temporal prediction in a periodic motion task. Frontiers in Human Neuroscience, 10, 268.

Cope, T. E., Grube, M., Singh, B., Burn, D. J., \& Griffiths, T. D. (2014). The basal ganglia in perceptual timing: Timing performance in multiple system atrophy and huntington's disease. Neuropsychologia, 52, 73-81.

Coull, J. T., Davranche, K., Nazarian, B., \& Vidal, F. (2013). Functional anatomy of timing differs for production versus prediction of time intervals. Neuropsychologia, 51, 309-319.

Coull, J. T., Frith, C. D., Büchel, C., \& Nobre, A. C. (2000). Orienting attention in time: Behavioural and neuroanatomical distinction between exogenous and endogenous shifts. Neuropsychologia, 38, 808-819.

Coull, J. T., \& Nobre, A. C. (1998). Where and when to pay attention: The neural systems for directing attention to spatial locations and to time intervals as revealed by both PET and fMRI. Journal of Neuroscience, 18, 7426-7435.

Coull, J. T., \& Nobre, A. (2008). Dissociating explicit timing from temporal expectation with fMRI. Current Opinion Neurobiology, 18, 137-144.

Coull, J. T., Vidal, F., Nazarian, B., \& Macar, F. (2004). Functional anatomy of the attentional modulation of time estimation. Science, 303, 1506-1508.

Craig, A. D. (2002). How do you feel? Interoception: The sense of the physiological condition of the body. Nature Reviews Neuroscience, 3, 655-666.

Craig, A. D. (2009a). Emotional moments across time: A possible neural basis for time perception in the anterior insula. Philosophical Transactions of the Royal Society of London: Series B: Biologucal Sciences, 364, 1933-1942.

Craig, A. D. (2009b). How do you feel-Now? The anterior insula and human awareness. Nature Reviews Neuroscience, $10,59-70$

Crick, F. C., \& Koch, C. (2005). What is the function of the claustrum? Philosophical Transactions of the Royal Society of London: Series B: Biologucal Sciences, 360, 1271-1279.

Critchley, H. D., Wiens, S., Rotshtein, P., Ohman, A., \& Dolan, R. J. (2004). Neural systems supporting interoceptive awareness. Nature Neuroscience, 7, 189-195.

Dirnberger, G., Hesselmann, G., Roiser, J. P., Preminger, S., Jahanshahi, M., \& Paz, R. (2012). Give it time: Neural evidence for distorted time perception and enhanced memory encoding in emotional situations. Neuroimage, 63, 591-599.

Dudukovic, N. M., \& Wagner, A. D. (2007). Goal-dependent modulation of declarative memory: Neural correlates of temporal recency decisions and novelty detection. Neuropsychologia , 45, 2608-2620.

Eickhoff, S. B., Bzdok, D., Laird, A. R., Kurth, F., \& Fox, P. T. (2012). Activation likelihood estimation meta-analysis revisited. Neuroimage, 59, 2349-2361.

Eickhoff, S. B., Laird, A. R., Fox, P. M., Lancaster, J. L., \& Fox, P. T. (2017). Implementation errors in the GingerALE 
software: Description and recommendations. Human Brain Mapping, 38, 7-11.

Eickhoff, S. B., Laird, A. R., Grefkes, C., Wang, L. E., Zilles, K., \& Fox, P. T. (2009). Coordinate-based activation likelihood estimation meta-analysis of neuroimaging data: A randomeffects approach based on empirical estimates of spatial uncertainty. Human Brain Mapping, 30, 2907-2926.

Eickhoff, S. B., Nichols, T. E., Laird, A. R., Hoffstaedter, F., Amunts, K., Fox, P. T., et al. (2016). Behavior, sensitivity, and power of activation likelihood estimation characterized by massive empirical simulation. Neuroimage, 137, 70-85.

Ferrandez, A. M., Hugueville, L., Lehéricy, S., Poline, J. B., Marsault, C., \& Pouthas, V. (2003). Basal ganglia and supplementary motor area subtend duration perception: An fMRI study. Neuroimage, 19, 1532-1544.

Fierro, B., Palermo, A., Puma, A., Francolini, M., Panetta, M. L., Daniele, O., et al. (2007). Role of the cerebellum in time perception: A TMS study in normal subjects. Journal of the Neurological Science, 263, 107-112.

Garraux, G., McKinney, C., Wu, T., Kansaku, K., Nolte, G., \& Hallett, M. (2005). Shared brain areas but not functional connections controlling movement timing and order. Journal of Neuroscience, 25, 5290-5297.

Gibbon, J., Malapani, C., Dale, C. L., \& Gallistel, C. (1997). Toward a neurobiology of temporal cognition: Advances and challenges. Current Opinion in Neurobiology, 7, 170-184.

Grube, M., Cooper, F. E., Chinnery, P. F., \& Griffiths, T. D. (2010). Dissociation of duration-based and beat-based auditory timing in cerebellar degeneration. Proceedings of the National Academy of Sciences, U.S.A., 107, 11597-11601.

Gutyrchik, E., Churan, J., Meindl, T., Bokde, A. L., von Bernewitz, H., Born, C., et al. (2010). Functional neuroimaging of duration discrimination on two different time scales. Neuroscience Letters, 469, 411-415.

Harrington, D. L., Boyd, L. A., Mayer, A. R., Sheltraw, D. M., Lee, R. R., Huang, M., et al. (2004). Neural representation of interval encoding and decision making. Brain Research: Cognitive Brain Research, 21, 193-205.

Harrington, D. L., \& Haaland, K. Y. (1999). Neural underpinnings of temporal processing: A review of focal lesion, pharmacological, and functional imaging research. Reviews in Neuroscience, 10, 91-116.

Harrington, D. L., Zimbelman, J. L., Hinton, S. C., \& Rao, S. M. (2010). Neural modulation of temporal encoding, maintenance, and decision processes. Cerebral Cortex, 20, 1274-1285.

Herrmann, B., Henry, M. J., Scharinger, M., \& Obleser, J. (2014). Supplementary motor area activations predict individual differences in temporal-change sensitivity and its illusory distortions. Neuroimage, 101, 370-379.

Hinton, S. C., Harrington, D. L., Binder, J. R., Durgerian, S., \& Rao, S. M. (2004). Neural systems supporting timing and chronometric counting: An fMRI study. Brain Research: Cognitive Brain Research, 21, 183-192.

Ivry, R. B. (1993). Cerebellar involvement in the explicit representation of temporal information. Annals of the New York Academy of Sciences, 682, 214-230.

Ivry, R. B., \& Keele, S. W. (1989). Timing functions of the cerebellum. Journal Cognitive Neuroscience, 1, 136-152.

Ivry, R. B., \& Schlerf, J. E. (2008). Dedicated and intrinsic models of time perception. Trends in Cognitive Sciences, 12, 273-280.

Ivry, R. B., Spencer, R. M., Zelaznik, H. N., \& Diedrichsen, J. (2002). The cerebellum and event timing. Annals of New York Academy of Science, 978, 302-317.

Jahanshahi, M., Jones, C. R., Dirnberger, G., \& Frith, C. D. (2006). The substantia nigra pars compacta and temporal processing. Journal of Neuroscience, 26, 12266-12273.
Jancke, L., Loose, R., Lutz, K., Specht, K., \& Shah, N. J. (2000). Cortical activations during paced finger-tapping applying visual and auditory pacing stimuli. Brain Research: Cognitive Brain Research, 10, 51-66.

Jantzen, K. J., Oullier, O., Marshall, M., Steinberg, F. L., \& Kelso, J. A. (2007). A parametric fMRI investigation of context effects in sensorimotor timing and coordination. Neuropsychologia, 45, 673-684.

Jantzen, K. J., Steinberg, F. L., \& Kelso, J. A. (2004). Brain networks underlying human timing behavior are influenced by prior context. Proceedings of the National Academy of Sciences, U.S.A., 101, 6815-6820.

Jantzen, K. J., Steinberg, F. L., \& Kelso, J. A. (2005). Functional MRI reveals the existence of modality and coordinationdependent timing networks. Neuroimage, 25, 1031-1042.

Jech, R., Dusek, P., Wackermann, J., \& Vymazal, J. (2005). Cumulative blood oxygenation-level-dependent signal changes support the 'time accumulator' hypothesis. NeuroReport, 16, 1467-1471.

Jueptner, M., Flerich, L., Weiller, C., Mueller, S. P., \& Diener, H. C. (1996). The human cerebellum and temporal information processing-Results from a PET experiment. NeuroReport, 7, 2761-2765.

Jueptner, M., Rijntjes, M., Weiller, C., Faiss, J. H., Timmann, D., Mueller, S. P., et al. (1995). Localization of a cerebellar timing process using PET. Neurology, 45, 1540-1545.

Karabanov, A., Blom, O., Forsman, L., \& Ullén, F. (2009). The dorsal auditory pathway is involved in performance of both visual and auditory rhythms. Neuroimage, 44, 480-488.

Kawashima, R., Okuda, J., Umetsu, A., Sugiura, M., Inoue, K., Suzuki, K., et al. (2000). Human cerebellum plays an important role in memory-timed finger movement: An fMRI study. Journal of Neurophysiology, 83, 1079-1087.

Knutson, K. M., Wood, J. N., \& Grafman, J. (2004). Brain activation in processing temporal sequence: An fMRI study. Neuroimage, 23, 1299-1307.

Koch, G., Oliveri, M., Torriero, S., Salerno, S., Lo Gerfo, E., \& Caltagirone, C. (2007). Repetitive TMS of cerebellum interferes with millisecond time processing. Experimental Brain Research, 179, 291-299.

Laird, A. R., Fox, P. M., Price, C. J., Glahn, D. C., Uecker, A. M., Lancaster, J. L., et al. (2005). ALE meta-analysis: Controlling the false discovery rate and performing statistical contrasts. Human Brain Mapping, 25, 155-164.

Laird, A. R., Robinson, J. L., McMillan, K. M., TordesillasGutiérrez, D., Moran, S. T., Gonzales, S. M., et al. (2010). Comparison of the disparity between Talairach and MNI coordinates in functional neuroimaging data: Validation of the Lancaster transform. Neuroimage, 51, 677-683.

Larsson, J., Gulyas, B., \& Roland, P. E. (1996). Cortical representation of self-paced finger movement. NeuroReport, 7, 463-468.

Lee, K. H., Egleston, P. N., Brown, W. H., Gregory, A. N., Barker, A. T., \& Woodruff, P. W. (2007). The role of the cerebellum in subsecond time perception: Evidence from repetitive transcranial magnetic stimulation. Journal Cognitive Neuroscience, 19, 147-157.

Lejeune, H., Maquet, P., Bonnet, M., Casini, L., Ferrara, A., Macar, F., et al. (1997). The basic pattern of activation in motor and sensory temporal tasks: Positron emission tomography data. Neuroscience Letters, 235, 21-24.

Leon, M. I., \& Shadlen, M. N. (2003). Representation of time by neurons in the posterior parietal cortex of the macaque. Neuron, 38, 317-327.

Lewis, P. A., \& Miall, R. C. (2002). Brain activity during nonautomatic motor production of discrete multi-second intervals. NeuroReport, 13, 1731-1735.

Lewis, P. A., \& Miall, R. C. (2003a). Distinct systems for automatic and cognitively controlled time measurement: 
Evidence from neuroimaging. Current Opinion Neurobiology, 13, 250-255.

Lewis, P. A., \& Miall, R. C. (2003b). Brain activation patterns during measurement of sub- and suprasecond intervals. Neuropsychologia , 41, 1583-1592.

Lewis, P. A., \& Miall, R. C. (2006a). Remembering the time: A continuous clock. Trends in Cognitive Sciences, 10, 401-406.

Lewis, P. A., \& Miall, R. C. (2006b). A right hemispheric prefrontal system for cognitive time measurement. Behavioural Processes, 71, 226-234.

Lewis, P. A., Wing, A. M., Pope, P. A., Praamstra, P., \& Miall, R. C. (2004). Brain activity correlates differentially with increasing temporal complexity of rhythms during initialisation, synchronisation, and continuation phases of paced finger tapping. Neuropsychologia, 42, 1301-1312.

Liberati, A., Altman, D. G., Tetzlaff, J., Mulrow, C., Gøtzsche, P. C., Ioannidis, J. P., et al. (2009). The PRISMA statement for reporting systematic reviews and meta-analyses of studies that evaluate health care interventions: Explanation and elaboration. Journal Clinical Epidemiology, 62, e1-e34.

Livesey, A. C., Wall, M. B., \& Smith, A. T. (2007). Time perception: Manipulation of task difficulty dissociates clock functions from other cognitive demands. Neuropsychologia, $45,321-331$.

Lutz, K., Specht, K., Shah, N. J., \& Jancke, L. (2000). Tapping movements according to regular and irregular visual timing signals investigated with fMRI. NeuroReport, 11, 1301-1306.

Lux, S., Marshall, J. C., Ritzl, A., Zilles, K., \& Fink, G. R. (2003). Neural mechanisms associated with attention to temporal synchrony versus spatial orientation: An fMRI study. Neuroimage, 20(Suppl. 1), S58-S65.

Macar, F., Anton, J. L., Bonnet, M., \& Vidal, F. (2004). Timing functions of the supplementary motor area: An event-related fMRI study. Brain Research: Cognitive Brain Research, 21, 206-215.

Macar, F., Coull, J., \& Vidal, F. (2006). The supplementary motor area in motor and perceptual time processing: fMRI studies. Cognitive Processing, 7, 89-94.

Macar, F., Lejeune, H., Bonnet, M., Ferrara, A., Pouthas, V., Vidal, F., et al. (2002). Activation of the supplementary motor area and of attentional networks during temporal processing. Experimental Brain Research, 142, 475-485.

Mancuso, L., Costa, T., Nani, A., Manuello, J., Liloia, D., Gelmini, G., et al. (2019). The homotopic connectivity of the functional brain: A meta-analytic approach. Scientic Reports, 9, 3346

Mangels, J. A., Ivry, R. B., \& Shimizu, N. (1998). Dissociable contributions of the prefrontal and neocerebellar cortex to time perception. Brain Research: Cognitive Brain Research, 7, 15-39.

Maquet, P., Lejeune, H., Pouthas, V., Bonnet, M., Casini, L., Macar, F., et al. (1996). Brain activation induced by estimation of duration: A PET study. Neuroimage, 3, 119-126.

Matell, M. S., \& Meck, W. H. (2000). Neuropsychological mechanisms of interval timing behavior. BioEssays, $22,94-103$

Matell, M. S., \& Meck, W. H. (2004). Cortico-striatal circuits and interval timing: Coincidence detection of oscillatory processes. Brain Research: Cognitive Brain Research, 21, 139-170.

Mathiak, K., Hertrich, I., Grodd, W., \& Ackermann, H. (2004). Discrimination of temporal information at the cerebellum: Functional magnetic resonance imaging of nonverbal auditory memory. Neuroimage, 21, 154-162.

Mathur, B. N. (2014). The claustrum in review. Frontiers in Systems Neuroscience, 8, 48.
Mauk, M. D., \& Buonomano, D. V. (2004). The neural basis of temporal processing. Annual Review of Neuroscience, 27, 307-340.

Mayville, J. M., Jantzen, K. J., Fuchs, A., Steinberg, F. L., \& Kelso, J. A. (2002). Cortical and subcortical networks underlying syncopated and synchronized coordination revealed using fMRI. Functional magnetic resonance imaging. Human Brain Mapping, 17, 214-229.

Meck, W. H. (2005). Neuropsychology of timing and time perception. Brain and Cognition, 58, 1-8.

Meck, W. H., Penney, T. B., \& Pouthas, V. (2008). Cortico-striatal representation of time in animals and humans. Current Opinion in Neurobiology, 18, 145-152.

Merchant, H., Harrington, D. L., \& Meck, W. H. (2013). Neural basis of the perception and estimation of time. Annual Review of Neuroscience, 36, 313-336.

Moher, D., Liberati, A., Tetzlaff, J., \& Altman, D. G. (2009). Preferred reporting items for systematic reviews and metaanalyses: The PRISMA statement. Journal of Clinical Epidemiology, 62, 1006-1012.

Morillon, B., Kell, C. A., \& Giraud, A. L. (2009). Three stages and four neural systems in time estimation. Journal of Neuroscience, 29, 14803-14811.

Murai, Y., \& Yotsumoto, Y. (2016). Context-dependent neural modulations in the perception of duration. Frontiers in Integrative Neuroscience, 10, 12.

Neufang, S., Fink, G. R., Herpertz-Dahlmann, B., Willmes, K., \& Konrad, K. (2008). Developmental changes in neural activation and psychophysiological interaction patterns of brain regions associated with interference control and time perception. Neuroimage, 43, 399-409.

Nobre, A. C., \& O'Reilly, J. (2004). Time is of the essence. Trends in Cognitive Sciences, 8, 387-389.

Oliveri, M., Koch, G., Salerno, S., Torriero, S., Lo Gerfo, E., \& Caltagirone, C. (2009). Representation of time intervals in the right posterior parietal cortex: Implications for a mental time line. Neuroimage, 46, 1173-1179.

Ortuño, F., Guillen-Grima, F., Lopez-Garcia, P., Gomez, J., \& Pla, J. (2011). Functional neural networks of time perception: Challenge and opportunity for schizophrenia research. Schizophrenia Research, 125, 129-135.

Ortuño, F., Ojeda, N., Arbizu, J., Lopez, P., Martí-Climent, J. M., Peñuelas, I., et al. (2002). Sustained attention in a counting task: Normal performance and functional neuroanatomy. Neuroimage, 17, 411-420.

Oullier, O., Jantzen, K. J., Steinberg, F. L., \& Kelso, J. A. (2005). Neural substrates of real and imagined sensorimotor coordination. Cerebral Cortex, 15, 975-985.

Pastor, M. A., Day, B. L., Macaluso, E., Friston, K. J., \& Frackowiak, R. S. (2004). The functional neuroanatomy of temporal discrimination. Journal of Neuroscience, 24, 2585-2591.

Penhune, V. B., Zattore, R. J., \& Evans, A. C. (1998). Cerebellar contributions to motor timing: A PET study of auditory and visual rhythm reproduction. Journal of Cognitive Neuroscience, 10, 752-765.

Pfeuty, M., Dilharreguy, B., Gerlier, L., \& Allard, M. (2015). fMRI identifies the right inferior frontal cortex as the brain region where time interval processing is altered by negative emotional arousal. Human Brain Mapping, 36, 981-995.

Pope, P., Wing, A. M., Praamstra, P., \& Miall, R. C. (2005). Force related activations in rhythmic sequence production. Neuroimage, 27, 909-918.

Pouthas, V., George, N., Poline, J. B., Pfeuty, M., Vandemoorteele, P. F., Hugueville, L., et al. (2005). Neural network involved in time perception: An fMRI study comparing long and short interval estimation. Human Brain Mapping, 25, 433-441. 
Pouthas, V., \& Perbal, S. (2004). Time perception depends on accurate clock mechanisms as well as unimpaired attention and memory processes. Acta Neurobiologiae Experimentalis, 64, 367-385.

Protopapa, F., Hayashi, M. J., Kulashekhar, S., van der Zwaag, W., Battistella, G., Murray, M. M., et al. (2019). Chronotopic maps in human supplementary motor area. PLOS BIOLOGY, 17, e3000026.

Radua, J., Del Pozo, N. O., Gomez, J., Guillen-Grima, F., \& Ortuño, F. (2014). Meta-analysis of functional neuroimaging studies indicates that an increase of cognitive difficulty during executive tasks engages brain regions associated with time perception. Neuropsychologia, 58, 14-22.

Rammsayer, T. H. (1999). Neuropharmacological evidence for different timing mechanisms in humans. The Quarterly Journal of Experimental Psychology: Comparative and Physiological Psychology, 52, 273-286.

Rao, S. M., Harrington, D. L., Haaland, K. Y., Bobholz, J. A., Cox, R. W., \& Binder, J. R. (1997). Distributed neural systems underlying the timing of movements. Journal of Neuroscience, 17, 5528-5535.

Rao, S. M., Mayer, A. R., \& Harrington, D. L. (2001). The evolution of brain activation during temporal processing. Nature Neuroscience, 4, 317-q323.

Rekkas, P. V., Westerveld, M., Skudlarski, P., Zumer, J., Pugh, K., Spencer, D. D., et al. (2005). Neural correlates of temporalorder judgments versus those of spatial-location: Deactivation of hippocampus may facilitate spatial performance. Brain and Cognition, 59, 103-113.

Riecker, A., Kassubek, J., Gröschel, K., Grodd, W., \& Ackermann, H. (2006). The cerebral control of speech tempo: Opposite relationship between speaking rate and BOLD signal changes at striatal and cerebellar structures. Neuroimage, 29, 46-53.

Riecker, A., Wildgruber, D., Mathiak, K., Grodd, W., \& Ackermann, H. (2003). Parametric analysis of rate-dependent hemodynamic response functions of cortical and subcortical brain structures during auditorily cued finger tapping: A fMRI study. Neuroimage, 18, 731-739.

Rosenthal, R. (1979). The file drawer problem and tolerance for null results. Psychological Bulletin, 86, 638-641.

Rovelli, C. (2018). The order of time. Penguin.

Rubia, K., Overmeyer, S., Taylor, E., Brammer, M., Williams, S., Simmons, A., et al. (1998). Prefrontal involvement in "temporal bridging" and timing movement. Neuropsychologia, 36, 1283-1293.

Sakai, K., Hikosaka, O., Miyauchi, S., Takino, R., Tamada, T., Iwata, N. K., et al. (1999). Neural representation of a rhythm depends on its interval ratio. Journal of Neuroscience, 19, 10074-10081.

Schubotz, R. I., Friederici, A. D., \& von Cramon, D. Y. (2000). Time perception and motor timing: A common cortical and subcortical basis revealed by fMRI. Neuroimage, 11, 1-12.

Schubotz, R. I., \& von Cramon, D. Y. (2001). Interval and ordinal properties of sequences are associated with distinct premotor areas. Cerebral Cortex, 11, 210-222.

Schwartze, M., Rothermich, K., \& Kotz, S. A. (2012). Functional dissociation of pre-SMA and SMA-proper in temporal processing. Neuroimage, 60, 290-298.

Seeley, W. W., Menon, V., Schatzberg, A. F., Keller, J., Glover, G. H., Kenna, H., et al. (2007). Dissociable intrinsic connectivity networks for salience processing and executive control. Journal of Neuroscience, 27, 2349-2356.

Shergill, S. S., Tracy, D. K., Seal, M., Rubia, K., \& McGuire, P. (2006). Timing of covert articulation: An fMRI study. Neuropsychologia, 44, 2573-2577.
Shih, L. Y., Kuo, W. J., Yeh, T. C., Tzeng, O. J., \& Hsieh, J. C. (2009). Common neural mechanisms for explicit timing in the subsecond range. NeuroReport, 20, 897-901.

Shih, L. Y., Yeh, T. C., Kuo, W. J., Tzeng, O. J., \& Hsieh, J. C. (2010). Effect of temporal difficulty on cerebrocerebellar interaction during visual duration discrimination. Behavioural Brain Research, 207, 155-160.

Singer, T., Critchley, H. D., \& Preuschoff, K. (2009). A common role of insula in feelings, empathy and uncertainty. Trends in Cognitive Sciences, 13, 334-340.

Smith, A., Taylor, E., Lidzba, K., \& Rubia, K. (2003). A right hemispheric frontocerebellar network for time discrimination of several hundreds of milliseconds. Neuroimage, 20, 344-350.

Smith, A. B., Giampietro, V., Brammer, M., Halari, R., Simmons, A., \& Rubia, K. (2011). Functional development of frontostriato-parietal networks associated with time perception. Frontiers in Human Neuroscience, 5, 136.

Smythies, J., Edelstein, L., \& Ramachandran, V. (2012). Hypotheses relating to the function of the claustrum. Frontiers in Integrative Neuroscience, 6, 53.

Smythies, J., Edelstein, L., \& Ramachandran, V. (2014). Hypotheses relating to the function of the claustrum II: Does the claustrum use frequency codes? Frontiers in Integrative Neuroscience, 8,7

Stevens, M. C., Kiehl, K. A., Pearlson, G., \& Calhoun, V. D. (2007). Functional neural circuits for mental timekeeping. Human Brain Mapping, 28, 394-408.

Taatgen, N. A., van Rijn, H., \& Anderson, J. (2007). An integrated theory of prospective time interval estimation: The role of cognition, attention, and learning. Psychological Review, 114, 577-598.

Taniwaki, T., Okayama, A., Yoshiura, T., Togao, O., Nakamura, Y., Yamasaki, T., et al. (2006). Functional network of the basal ganglia and cerebellar motor loops in vivo: Different activation patterns between self-initiated and externally triggered movements. Neuroimage, 31, $745-753$.

Teki, S. (2016). A citation-based analysis and review of significant papers on timing and time perception. Frontiers in Neuroscience, 10, 330.

Teki, S., \& Griffiths, T. D. (2016). Brain bases of working memory for time intervals in rhythmic sequences. Frontiers in Neuroscience, 10, 239.

Tipples, J., Brattan, V., \& Johnston, P. (2013). Neural bases for individual differences in the subjective experience of short durations (less than 2 seconds). PLOS ONE, 8, e54669.

Tomasi, D., Wang, G. J., Studentsova, Y., \& Volkow, N. D. (2015). Dissecting neural responses to temporal prediction, attention, and memory: Effects of reward learning and interoception on time perception. Cerebral Cortex, 25, 3856-3867.

Torgerson, C. M., \& Van Horn, J. D. (2014). A case study in connectomics: The history, mapping, and connectivity of the claustrum. Frontiers in Neuroinformatics, 8, 83.

Tracy, J. I., Faro, S. H., Mohamed, F. B., Pinsk, M., \& Pinus, A. (2000). Functional localization of a "Time Keeper" function separate from attentional resources and task strategy. Neuroimage, 11, 228-242.

Tregellas, J. R., Davalos, D. B., \& Rojas, D. C. (2006). Effect of task difficulty on the functional anatomy of temporal processing. Neuroimage, 32, 307-315.

Tsukamoto, T., Kotani, Y., Ohgami, Y., Omura, K., Inoue, Y., \& Aihara, Y. (2006). Activation of insular cortex and subcortical regions related to feedback stimuli in a time estimation task: An fMRI study. Neuroscience Letters, 399, 39-44. 
Turkeltaub, P. E., Eickhoff, S. B., Laird, A. R., Fox, M., Wiener, M., \& Fox, P. (2012). Minimizing within-experiment and within-group effects in activation likelihood estimation metaanalyses. Human Brain Mapping, 33, 1-13.

Tzourio, N., Massioui, F. E., Crivello, F., Joliot, M., Renault, B., \& Mazoyer, B. (1997). Functional anatomy of human auditory attention studied with PET. Neuroimage, 5, 63-77.

Unger, R. M., \& Smolin, L. (2015). The singular universe and the reality of time. Cambridge University Press.

Ustun, S., Kale, E. H., \& Cicek, M. (2017). Neural networks for time perception and working memory. Frontiers Human Neuroscience, 11, 83.

van Wassenhove, V., Wittmann, M., Craig, A. D., \& Paulus, M. P. (2011). Psychological and neural mechanisms of subjective time dilation. Frontiers Neuroscience, 5, 56.

Vicario, C. M., Martino, D., \& Koch, G. (2013). Temporal accuracy and variability in the left and right posterior parietal cortex. Neuroscience, 245, 121-128.

Westheimer, G. (1999). Discrimination of short time intervals by the human observer. Experimental Brain Reserach, 129, 121-126.

Wiener, M., Lee, Y. S., Lohoff, F. W., \& Coslett, H. B. (2014). Individual differences in the morphometry and activation of time perception networks are influenced by dopamine genotype. Neuroimage, 89, 10-22.

Wiener, M., Turkeltaub, P., \& Coslett, H. B. (2010). The image of time: A voxel-wise meta-analysis. Neuroimage, 49, 1728-1740.

Wittmann, M. (2013). The inner sense of time: How the brain creates a representation of duration. Nature Reviews Neuroscience, 14, 217-223.

Wittmann, M., Simmons, A. N., Aron, J. L., \& Paulus, M. P. (2010). Accumulation of neural activity in the posterior insula encodes the passage of time. Neuropsychologia , 48, 3110-3120.

Wittmann, M., Simmons, A. N., Flagan, T., Lane, S. D., Wackermann, J., \& Paulus, M. P. (2011). Neural substrates of time perception and impulsivity. Brain Research, 1406, 43-58.

Wittmann, M., \& van Wassenhove, V. (2009). The experience of time: Neural mechanisms and the interplay of emotion, cognition and embodiment. Philosophical Transantactions of the Royal Society of London: Series B: Biological Sciences, 364, 1809-1813.

Wittmann, M., van Wassenhove, V., Craig, A. D., \& Paulus, M. P. (2010). The neural substrates of subjective time dilation. Frontiers in Human Neuroscience, 4, 2.

Zakay, D., \& Block, R. A. (1997). Temporal cognition. Current Directions in Psychological Science, 6, 12-16. 